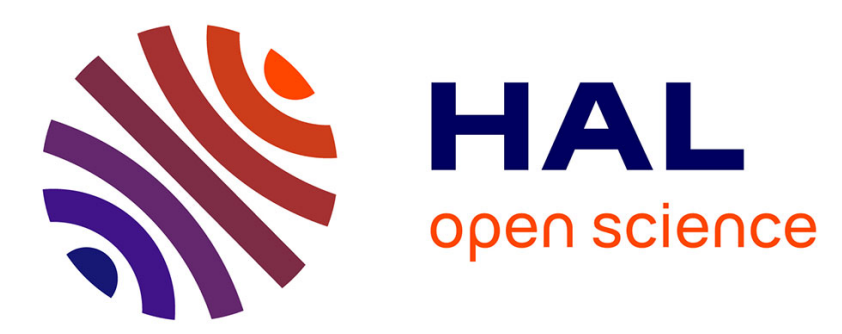

\title{
Stability of Perfectly Matched Layers, Group Velocities and Anisotropic Waves
}

Eliane Bécache, Sandrine Fauqueux, Patrick Joly

\section{To cite this version:}

Eliane Bécache, Sandrine Fauqueux, Patrick Joly. Stability of Perfectly Matched Layers, Group Velocities and Anisotropic Waves. [Research Report] RR-4304, INRIA. 2001. inria-00072283

\section{HAL Id: inria-00072283 \\ https://hal.inria.fr/inria-00072283}

Submitted on 23 May 2006

HAL is a multi-disciplinary open access archive for the deposit and dissemination of scientific research documents, whether they are published or not. The documents may come from teaching and research institutions in France or abroad, or from public or private research centers.
L'archive ouverte pluridisciplinaire HAL, est destinée au dépôt et à la diffusion de documents scientifiques de niveau recherche, publiés ou non, émanant des établissements d'enseignement et de recherche français ou étrangers, des laboratoires publics ou privés. 


\section{Stability of Perfectly Matched Layers, Group Velocities and Anisotropic Waves}

Eliane BECACHE, Sandrine FAUQUEUX, Patrick JOLY

\section{$\mathbf{N}^{\circ} 4304$}

Novembre 2001

THÈME 4 



\title{
Stability of Perfectly Matched Layers, Group Velocities and Anisotropic Waves
}

\author{
Eliane BECACHE*, Sandrine FAUQUEUX ${ }^{\dagger}$, Patrick JOLY ${ }^{\ddagger}$ \\ Thème 4 - Simulation et optimisation \\ de systèmes complexes \\ Projet Ondes
}

Rapport de recherche $n^{\circ} 4304$ - Novembre 2001 - 35 pages

\begin{abstract}
Perfectly Matched Layers (PML) are a recent technique for simulating the absorption of waves in open domains. They have been introduced for electromagnetic waves and extended, since then, to other models of wave propagation, including waves in elastic anisotropic media. In this last case, some numerical experiments have shown that the PMLs are not always stable. In this paper, we investigate this question from a theoretical point of view. In the first part, we derive a necessary condition for the stability of the PML model for a general hyperbolic system. This condition can be interpreted in terms of geometrical properties of the slowness diagrams and used for explaining instabilities observed with elastic waves but also with other propagation models (anisotropic Maxwell's equations, linearized Euler equations). In the second part, we specialize our analysis to orthotropic elastic waves and obtain separately a necessary stability condition and a sufficient stability condition that can be expressed in terms of inequalities on the elasticity coefficients of the model.
\end{abstract}

Key-words: perfectly matched layers, absorbing layers, elastodynamics, stability, hyperbolic systems, Fourier analysis, linearized Euler equations, anisotropy

\footnotetext{
* INRIA-rocquencourt. Eliane.Becache@inria.fr

† IFP, 1 avenue Bois Préau, 92500 Reuil Malmaison, et INRIA-rocquencourt. Sandrine.Fauqueux@inria.fr

$\ddagger$ INRIA-rocquencourt. Patrick.Joly@inria.fr
} 


\section{Stabilité des PML, vitesses de groupe et ondes anisotropes}

Résumé : Les couches parfaitement adaptées (PML) sont une technique récente pour simuler l'absorption des ondes dans des domaines non bornés. Elles ont été introduites pour les ondes électromagnétiques et depuis étendues à d'autres modèles de propagation d'ondes, notamment la propagation d'ondes élastiques en milieu anisotrope. Dans ce dernier cas, des simulations numériques ont montré que les PML ne sont pas toujours stables. Dans ce travail, nous nous intéressons à cette question d'un point de vue théorique. Dans la première partie, nous établissons une condition nécessaire de stabilité pour un système hyperbolique général. Cette condition peut être interprétée en terme de propriétés géométriques des surfaces de lenteur et utilisée pour expliquer des instabilités observées avec les ondes élastiques mais aussi avec d'autres modèles de propagation (équations de Maxwell anisotropes, équations d'Euler linéarisées). Dans la deuxième partie, nous concentrons notre analyse aux ondes élastiques orthotropes et obtenons séparément une condition nécessaire de stabilité et une condition suffisante de stabilité qui peuvent être exprimées comme des inégalités sur les coefficients élastiques du modèle.

Mots-clés : couches parfaitement adaptées, couches absorbantes, élastodynamique, stabilité, systèmes hyperboliques, analyse de Fourier, équations d'Euler linéarisées, anisotropie 


\section{Table of Contents}

1 Introduction $\quad 3$

2 Orthotropic elastic waves and PML model $\quad 4$

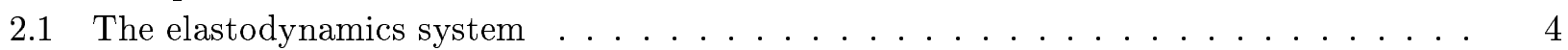

2.2 Harmonic plane waves and slowness diagrams . . . . . . . . . . . . . . . . 5

2.3 The PML model for a general first-order evolution problem . . . . . . . . . . 8

2.4 The PML Model for elastodynamics . . . . . . . . . . . . . . . . . . . 9

2.5 Some instructive numerical simulations . . . . . . . . . . . . . . . . . . 10

3 A necessary stability condition for PML models associated to general hyperbolic system. Interpretation in terms of slowness curves. 14

3.1 Well-posedness and stability: definition and characterization through plane wave analysis 14

3.2 A general well-posedness result . . . . . . . . . . . . . . . . 16

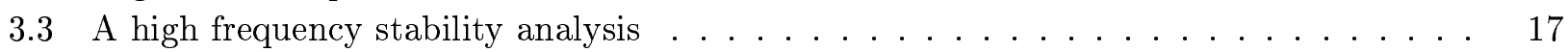

3.4 Application of the geometrical stability criterion . . . . . . . . . . . . 19

3.4.1 Explanation of the observations of section 2.5 via the analysis of slowness diagrams. 19

3.4.2 Application to other mathematical models . . . . . . . . . . . . . . 20

3.4.3 Other instability phenomena via numerical simulations . . . . . . . . . . . 22

4 Stability analysis for the orthotropic elastic model 24

4.1 A first high frequency necessary stability condition . . . . . . . . . . . . . . . 26

4.2 A second high frequency necessary stability condition . . . . . . . . . . . . . . . 28

4.3 A sufficient stability condition . . . . . . . . . . . . . . . . . 29

4.4 Back to the numerical experiments of section $3.4 .3 \ldots \ldots \ldots \ldots$

\section{Introduction}

Perfectly Matched Layers (PML) are a recent technique for simulating the absorption of waves in open domains, which provides a very efficient alternative to the use of absorbing boundary conditions in many applications. The idea is to surround the computational domain with an absorbing layer (the PML region) and the coupled system possesses the property of generating no reflection at the interface between the free medium and the artificial absorbing medium. This technique has been initially introduced by Bérenger [4] for Maxwell's equations, and has been widely used for the simulation of time dependent electromagnetic waves as well as Helmholtz-like equations (e.g., [5, 6, 25, 22, 23]). The method has been extended to various propagation models (the paraxial wave equations [9], the linearized Euler equations $[21,15,14], \ldots)$, including in particular elastic wave propagation in isotropic [13] and anisotropic media [11]. Trying to use this PMLs for computing the propagation of seismic waves, one of the authors observed blow up phenomena in some numerical experiments involving anisotropic media. Analogous instabilities have also been observed for the simulation of non destructive testing experiments [12]. This has motivated us to look at the question of the well-posedness and the stronger notion of stability of PMLs for anisotropic elastic waves from a theoretical point of view. It is important here to make precise the distinction (see section 3) between these two notions that are often identified in the literature. As a matter of fact, if the difference is fundamental from the mathematical point of view, for practical applications, it is not, and the good concept is the one of stability (in particular for absorbing waves). This type of mathematical questions has already been widely investigated by several authors $[24,18,17,19,3]$ in the case of Maxwell's equations. For elastodynamics equations, it is easy to show that the PML model is well-posed (cf section 3.2) but we will show that it can be unstable. The outline of this paper is as follows.

$\mathrm{RR} \mathrm{n}^{\circ} 4304$ 
- In section 2, we recall the propagation equations for elastic waves in a $2 \mathrm{D}$ orthotropic medium (section 2.1) and recall the important notions of slowness diagrams (section 2.2). In section 2.3, we present the construction of the standard PML model for a general first order hyperbolic system and specialize it to elastic waves in section 2.4. Finally section 2.5 is devoted to various simulations that point out the instability phenomena that motivate the present works.

- In section 3 we derive a necessary condition for the stability of the PML model for a general hyperbolic system (theorem 1). This condition can be interpreted in terms of geometrical properties of the slowness diagrams and used for explaining instabilities observed with elastic waves but also with other propagation models (anisotropic Maxwell's equations, linearized Euler equations). Finally we present numerical simulations showing that this geometrical criterium is not sufficient for stability in the case of elastic waves.

- In section 4, we specialize our analysis to orthotropic elastic waves and obtain separately a necessary stability condition (theorem 3 ) and a sufficient stability condition that can be expressed in terms of inequalities on the elasticity coefficients of the model (theorem 4).

\section{Orthotropic elastic waves and PML model}

\subsection{The elastodynamics system}

In this article we work in two dimensions. All the indices $i, j, k, l$ take their values in $\{1,2\} . x=\left(x_{1}, x_{2}\right)$ denotes the space variable and $\partial_{i}$ holds for the derivation with respect to $x_{i}$. Throughout this section, we shall use the Einstein convention on repeated indices for summation. Let us introduce the equations of elastodynamics in a $2 \mathrm{D}$ homogeneous medium:

$$
\rho \frac{\partial^{2} u}{\partial t^{2}}-\operatorname{div} \sigma(u)=0
$$

where $u=\left(u_{i}\right)$ denotes the displacement field, $\rho>0$ the density, $\sigma(u)=\sigma_{i j}(u)$ the stress tensor and:

$$
\operatorname{div} \sigma=\left(\begin{array}{c}
\partial_{1} \sigma_{11}+\partial_{2} \sigma_{12} \\
\partial_{1} \sigma_{12}+\partial_{2} \sigma_{22}
\end{array}\right)
$$

The stress tensor is related to the strain tensor $\varepsilon(u)$ defined by

$$
\varepsilon_{i j}(u)=\frac{1}{2}\left(\partial_{i} u_{j}+\partial_{j} u_{i}\right)
$$

by Hooke's law:

$$
A \sigma=\varepsilon(u) \Longleftrightarrow \sigma=C \varepsilon(u)=A^{-1} \varepsilon(u)
$$

The fourth-order tensor $C$ of elasticity coefficients satisfies the classical symmetries, [2]:

$$
C_{i j k l}=C_{k l i j}=C_{j i k l} .
$$

Moreover, it is positive definite:

$$
C_{i j k l} \xi_{i j} \xi_{k l} \geq \alpha\|\xi\|^{2}=\alpha \xi_{i j} \xi_{i j}
$$

for all symmetric tensor $\xi$. 
Notation. As it is classical, the symmetries (4) of the tensor $C$ allows us to simplify its representation into a $3 \times 3$ matrix $c_{p q}$, still denoted by $C$ for simplicity, such that

$$
C_{i j k l}=c_{p(i, j), p(k, l)}
$$

where the function $p$ is defined by:

$$
p(1,1)=1, \quad p(2,2)=2, \quad p(1,2)=p(2,1)=3 .
$$

Orthotropic media. In an orthotropic medium whose principal axes coincides with the $(x, y)$ axes, we have $c_{13}=c_{23}=0$ so that [2]:

$$
C=\left(\begin{array}{ccc}
c_{11} & c_{12} & 0 \\
c_{12} & c_{22} & 0 \\
0 & 0 & c_{33}
\end{array}\right)
$$

With this notation, the condition (5) becomes (|.| denotes the Euclidean norm in $\mathbb{R}^{3}$ ):

$$
c_{11} V_{1}^{2}+c_{22} V_{2}^{2}+2 c_{12} V_{1} V_{2}+c_{33} V_{3}^{2}>\alpha|V|^{2}, \quad \forall V \in \mathbb{R}^{3},
$$

which is equivalent to the inequalities:

$$
c_{11}>0, \quad c_{22}>0, \quad c_{33}>0, \quad c_{11} c_{22}-c_{12}^{2}>0 .
$$

For an isotropic medium, which is of course a particular orthotropic material, the coefficients can be expressed in terms of Lamé's coefficients $\lambda>0$ and $\mu \geq 0$ :

$$
c_{11}=c_{22}=\lambda+2 \mu, c_{12}=\lambda, c_{33}=\mu .
$$

In order to apply the general construction of the PML model, we will need to reformulate the propagation equations as a first-order system, the so-called velocity-stress formulation:

$$
\left\{\begin{array}{c}
\rho \frac{\partial v}{\partial t}-\operatorname{div} \sigma=0 \\
A \frac{\partial \sigma}{\partial t}-\varepsilon(v)=0
\end{array}\right.
$$

where $v=\partial_{t} u$ is the velocity field.

\subsection{Harmonic plane waves and slowness diagrams}

Plane waves are particular solutions of (1) on the form:

$$
u(x, t)=D e^{i(\omega t-k \cdot x)}, \quad D \in \mathbb{R}^{2}, \quad x=\left(x_{1}, x_{2}\right)
$$

where $k \in \mathbb{R}^{2}$ is the wave vector, $\omega \in \mathbb{R}$ the circular frequency and $D$ the displacement vector (or polarization vector). We also introduce

$$
\mid \begin{array}{ll}
K=\frac{k}{|k|}, & \text { the unit propagation direction, } \\
\mathcal{V}=\frac{\omega}{|k|}, & \text { the phase velocity, } \\
\overrightarrow{\mathcal{S}}=\frac{k}{\omega}, & \text { the slowness vector. }
\end{array}
$$


Plane waves of the form (10) are solutions of (1) if the circular frequency and the wave vector $k$ satisfy a relation, called dispersion relation. In order to write this relation, it is useful to introduce the Chrystoffel's tensor, [2]:

$$
\Gamma_{i j}(k)=C_{i l j m} k_{l} k_{m}
$$

which can be rewritten with the new notation:

$$
\Gamma(k)=\left(\begin{array}{cc}
c_{11} k_{1}^{2}+2 c_{13} k_{1} k_{2}+c_{33} k_{2}^{2} & c_{13} k_{1}^{2}+\left(c_{12}+c_{33}\right) k_{1} k_{2}+c_{32} k_{2}^{2} \\
c_{13} k_{1}^{2}+\left(c_{12}+c_{33}\right) k_{1} k_{2}+c_{32} k_{2}^{2} & c_{33} k_{1}^{2}+2 c_{23} k_{1} k_{2}+c_{22} k_{2}^{2}
\end{array}\right) .
$$

So, in an orthotropic medium, we have:

$$
\Gamma(k)=\left(\begin{array}{cc}
c_{11} k_{1}^{2}+c_{33} k_{2}^{2} & \left(c_{12}+c_{33}\right) k_{1} k_{2} \\
\left(c_{12}+c_{33}\right) k_{1} k_{2} & c_{33} k_{1}^{2}+c_{22} k_{2}^{2}
\end{array}\right) .
$$

We notice that $\Gamma(k)$ is homogeneous of degree 2 with respect to $k$ :

$$
\Gamma(\alpha k)=\alpha^{2} \Gamma(k) .
$$

It is well known that, due to (5), for any $k \neq 0, \Gamma(k)$ is symmetric positive definite [2]. Substituting (10) into (1), one sees that, the polarization vector $D$ must be an eigenvector of $\Gamma(k)$ associated to the eigenvalue $\rho \omega^{2}$ :

$$
\Gamma(k) D(k)=\rho \omega^{2} D(k) \Longleftrightarrow \Gamma(K) D=\rho \mathcal{V}^{2} D
$$

The frequency $\omega$ and the wave vector $k$ are thus related by the following dispersion relation:

$$
F(\omega, k) \equiv \operatorname{det}\left(\Gamma(k)-\rho \omega^{2} I\right)=0
$$

where $I$ is the identity matrix. Considering (15) as an equation in the frequency $\omega$ for a given value of the wave vector $k$, we obtain four solutions:

$$
\omega= \pm \omega_{Q P}(k), \quad \omega= \pm \omega_{Q S}(k), \quad\left(\omega_{Q P}(k)>0, \quad \omega_{Q S}(k)>0\right)
$$

where $\gamma_{Q P}(k)=\rho \omega_{Q P}^{2}(k) \geq \gamma_{Q P}(k)=\rho \omega_{Q S}^{2}(k)$ are the two positive eigenvalues of $\Gamma(k)$ associated to the respective eigenvectors $D_{Q P}(k)$ and $D_{Q S}(k)$,

- For $\omega= \pm \omega_{Q P}(k)$ and $D / / D_{Q P}(k)$, the wave is called quasi-longitudinal,

- For $\omega= \pm \omega_{Q S}(k)$ and $D / / D_{Q S}(k)$, the wave is called quasi-transverse.

The expressions of the eigenfunctions are:

$$
\left\{\begin{array}{c}
\gamma_{Q P}(k)=\frac{1}{2}\left(\Gamma_{11}(k)+\Gamma_{22}(k)+\sqrt{\left(\Gamma_{11}(k)-\Gamma_{22}(k)\right)^{2}+4 \Gamma_{12}(k)^{2}}\right) \\
\gamma_{Q S}(k)=\frac{1}{2}\left(\Gamma_{11}(k)+\Gamma_{22}(k)-\sqrt{\left(\Gamma_{11}(k)-\Gamma_{22}(k)\right)^{2}+4 \Gamma_{12}(k)^{2}}\right)
\end{array}\right.
$$

In particular in an isotropic medium, we recover the usual pressure and shear waves:

- $\omega_{Q P}(k)=|k| \mathcal{V}_{P}, \quad \mathcal{V}_{P}=\sqrt{\frac{\lambda+2 \mu}{\rho}}, \quad\left(\right.$ in this case $\left.D^{Q P}(k) / / k\right)$

- $\omega_{Q S}(k)=|k| \mathcal{V}_{S}, \quad \mathcal{V}_{S}=\sqrt{\frac{\mu}{\rho}}, \quad$ (in this case $\left.D^{Q S}(k) \perp k\right)$

INRIA 
Slowness diagram and group velocity. By homogeneity, the dispersion relation (15) can be rewritten as:

$$
F\left(1, \frac{k}{\omega}\right)=F(1, \vec{S})=0 .
$$

By definition, the slowness diagram is the set of points, in the plane of slowness vectors $\vec{S}=k / \omega$, that satisfy (17). It is also the union of two curves whose equations in polar coordinates are

$$
|\vec{S}|=\frac{1}{\omega_{Q P}(K)} \quad \text { and } \quad|\vec{S}|=\frac{1}{\omega_{Q S}(K)} .
$$
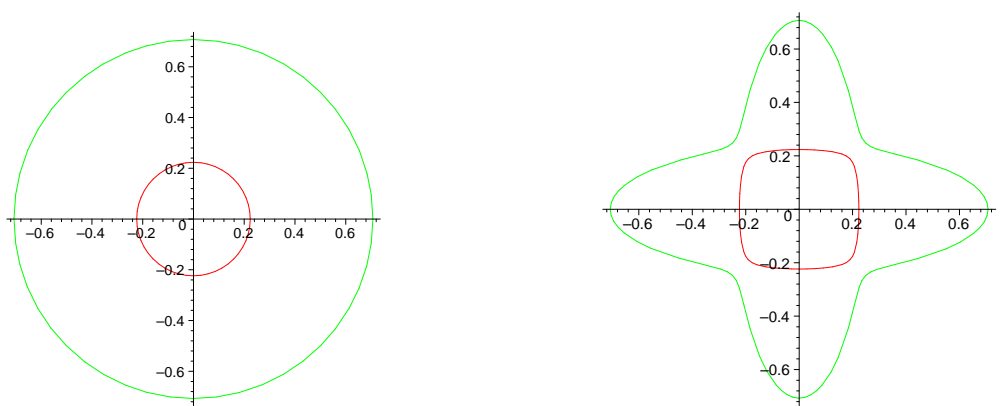

Figure 1: Slowness diagrams. Left: isotropic medium. Right: orthotropic medium.

We give in Figure 1 two examples of slowness diagrams. On the left, for an isotropic medium (the two curves are two circles respectively of radius $1 / \mathcal{V}_{P}$ and $\left.1 / \mathcal{V}_{S}>1 / \mathcal{V}_{P}\right)$ and on the right for an anisotropic medium $\left(c_{11}=c_{22}=20, c_{33}=2, c_{12}=3.8\right)$.

For each branch of solution $k \rightarrow \omega(k)$ of the dispersion relation, one define the group velocity as follows:

$$
\vec{V}_{g}(k)=\nabla_{k} \omega(k)
$$

Using (15), by implicit function theorem, it is easy to see that the group velocity can also be expressed as:

$$
\vec{V}_{g}(k)=-\left(\frac{\partial F}{\partial \omega}(\omega, k)\right)^{-1} \nabla_{k} F(\omega, k)=-\left(\frac{\partial F}{\partial \omega}(1, \vec{S})\right)^{-1} \nabla_{k} F(1, \vec{S})
$$

which shows that the group velocity is orthogonal to the slowness curves. Finally, the wave fronts are defined as the curves described by the extremities of the group velocities. These curves permit to predict the localization of the energy of a solution emitted from a point source (see Fig. 2).
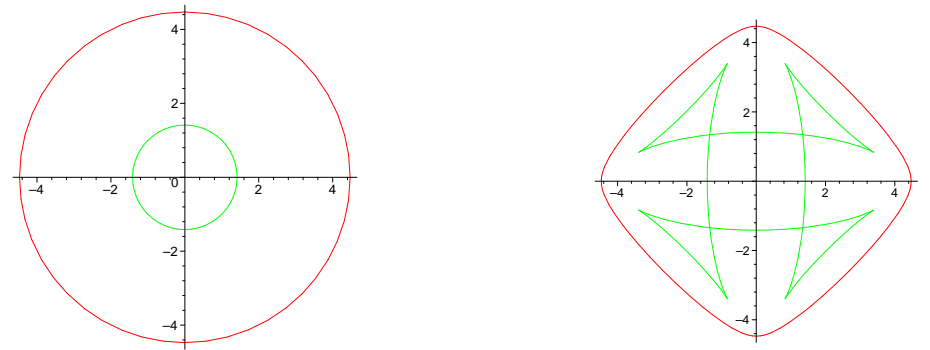

Figure 2: Waves fronts. Left: isotropic medium. Right: orthotropic medium. 


\subsection{The PML model for a general first-order evolution problem}

The principle of the perfectly matched layers has been first introduced by Bérenger [4], for electromagnetic waves and has been, since then, applied in numerous applications. In [11], the authors have shown that it is possible to write a systematic way of designing a PML model for a general first-order hyperbolic system. In this section we briefly describe this general construction. Consider a general first order hyperbolic system, posed initially in $\mathbb{R}^{m}$, of the form

$$
\partial_{t} u-A_{1} \partial_{1} u-A_{2} \partial_{2} u=0
$$

where $u$ is a m-vector, $A_{1}$ and $A_{2}$ are $m \times m$ matrices.

Let us briefly recall the definitions of hyperbolicity (see [16]). We set $\mathcal{A}(k)=k_{1} A_{1}+k_{2} A_{2}$. The system (20) is hyperbolic if, for all $k \in \mathbb{R}^{2}$, the eigenvalues of $\mathcal{A}(k)$ are real. It is strongly hyperbolic if furthermore, for all $k \in \mathbb{R}^{2}, \mathcal{A}(k)$ can be diagonalized (if not it is weakly hyperbolic). It is strictly hyperbolic if, for all $k \in \mathbb{R}^{2}$, the eigenvalues of $\mathcal{A}(k)$ are real and distinct.

The formal construction of a PML in the $x_{1}$ direction (parallel to the $x_{2}$ axis) consists in: (i) introducing a splitting of the field $u=u^{1}+u^{2}$, such that the unknown $u^{1}$ is only "associated" to the derivatives with respect to $x_{1}$, and $u^{2}$ to the derivatives with respect to $x_{2}$, (ii) introducing an anisotropic damping factor only on the $u^{1}$ component. We then obtain the following PML system:

$$
\left\{\begin{array}{l}
\partial_{t} u^{1}+\zeta\left(x_{1}\right) u^{1}-A_{1} \partial_{1}\left(u^{1}+u^{2}\right)=0 \\
\partial_{t} u^{2}-A_{2} \partial_{2}\left(u^{1}+u^{2}\right)=0
\end{array}\right.
$$

where $\zeta\left(x_{1}\right)=0$ for $x_{1}<0$ and $\zeta\left(x_{1}\right) \geq 0$ for $x_{1} \geq 0$.

In the frequency domain, the system (21) becomes:

$$
\left\{\begin{array}{l}
i \omega u^{1}+\zeta\left(x_{1}\right) u^{1}-A_{1} \partial_{1}\left(u^{1}+u^{2}\right)=0, \\
i \omega u^{2}-A_{2} \partial_{2}\left(u^{1}+u^{2}\right)=0
\end{array}\right.
$$

which can be obtained by substituting in the equation (20), written in the frequency domain, the $x_{1}$ derivatives $\partial_{1}$ into $d\left(\omega, \zeta\left(x_{1}\right)\right) \partial_{1}$, (see $[11,10]$ ), where:

$$
d\left(\omega, \zeta\left(x_{1}\right)\right)=\frac{i \omega}{\zeta\left(x_{1}\right)+i \omega}
$$

Actually, this corresponds to apply the complex change of variable [10, 20,7]:

$$
x_{1} \rightarrow x_{1}+\frac{1}{i \omega} \int_{0}^{x_{1}} \zeta(\xi) d \xi
$$

to the solution of the frequency domain version of (20). The very astonishing property of this layer model is that it is perfectly matched, which means that it generates no reflection at the interface between the physical domain and the absorbing medium (see [11]). This property can be shown through a plane wave analysis. Furthermore, this analysis also shows that the transmitted wave decreases exponentially during its propagation inside the layer.

Of course to construct the PML model in the $x_{2}$ direction it suffices to permute the role of the space directions which gives the following system:

$$
\left\{\begin{array}{l}
\partial_{t} u^{1}+u^{1}-A_{1} \partial_{1}\left(u^{1}+u^{2}\right)=0 \\
\partial_{t} u^{2}+\zeta\left(x_{2}\right) u^{2}-A_{2} \partial_{2}\left(u^{1}+u^{2}\right)=0
\end{array}\right.
$$




\subsection{The PML Model for elastodynamics}

It is then straightforward to obtain the PML model for the elastodynamics system (9), that can be rewritten as:

$$
\left\{\begin{array}{l}
\rho \frac{\partial v}{\partial t}-D^{1} \frac{\partial \sigma}{\partial x}-D^{2} \frac{\partial \sigma}{\partial y}=0 \\
A \frac{\partial \sigma}{\partial t}-E^{1} \frac{\partial v}{\partial x}-E^{2} \frac{\partial v}{\partial y}=0
\end{array}\right.
$$

where

$$
D^{1}=\left(\begin{array}{lll}
1 & 0 & 0 \\
0 & 0 & 1
\end{array}\right), \quad D^{2}=\left(\begin{array}{lll}
0 & 0 & 1 \\
0 & 1 & 0
\end{array}\right), \quad E^{1}=\left(\begin{array}{lll}
1 & 0 & 0 \\
0 & 0 & 1 / 2
\end{array}\right)^{t}, \quad E^{2}=\left(\begin{array}{lll}
0 & 0 & 1 / 2 \\
0 & 1 & 0
\end{array}\right)^{t}
$$

The PML system is then obtained by splitting $\sigma=\sigma^{1}+\sigma^{2}$ and $v=v^{1}+v^{2}$ (see [11]):

$$
\left\{\begin{array}{l}
\rho \frac{\partial v^{1}}{\partial t}+\zeta(x) v^{1}-D^{1} \frac{\partial}{\partial x}\left(\sigma^{1}+\sigma^{2}\right)=0 \\
\rho \frac{\partial v^{2}}{\partial t}-D^{2} \frac{\partial}{\partial y}\left(\sigma^{1}+\sigma^{2}\right)=0 \\
A \frac{\partial \sigma^{1}}{\partial t}+\zeta(x) \sigma^{1}-E^{1} \frac{\partial}{\partial x}\left(v^{1}+v^{2}\right)=0 \\
A \frac{\partial \sigma^{2}}{\partial t}-E^{2} \frac{\partial}{\partial y}\left(v^{1}+v^{2}\right)=0
\end{array}\right.
$$

In the frequency domain, this system can be written in $u$ as:

$$
\left\{\begin{array}{l}
\rho \omega^{2} u+\operatorname{div}_{p m l} \sigma(u)=0 \\
\sigma(u)=C \varepsilon_{p m l}(u)
\end{array}\right.
$$

which is the same model as in the physical domain, substituting the operators $\operatorname{div}$ and $\varepsilon$ with $\operatorname{div}_{p m l}$ and $\varepsilon_{p m l}$, where:

$$
\begin{gathered}
\operatorname{div}_{p m l} \sigma=\left(\begin{array}{cc}
d\left(\omega, \zeta\left(x_{1}\right)\right) & \partial_{1} \sigma_{11}+\partial_{2} \sigma_{12} \\
d\left(\omega, \zeta\left(x_{1}\right)\right) & \partial_{1} \sigma_{12}+\partial_{2} \sigma_{22}
\end{array}\right) \\
\varepsilon_{p m l}(u)=\left(\begin{array}{cc}
d\left(\omega, \zeta\left(x_{1}\right)\right) \partial_{1} u_{1} & \frac{1}{2}\left(d\left(\omega, \zeta\left(x_{1}\right)\right) \partial_{1} u_{2}+\partial_{2} u_{1}\right) \\
\frac{1}{2}\left(d\left(\omega, \zeta\left(x_{1}\right)\right) \partial_{1} u_{2}+\partial_{2} u_{1}\right) & \partial_{2} u_{2}
\end{array}\right) .
\end{gathered}
$$

The PML model in the $x_{2}$ direction still has the general form (26) but this time:

$$
\begin{gathered}
\operatorname{div}_{p m l} \sigma=\left(\begin{array}{c}
\partial_{1} \sigma_{11}+d\left(\omega, \zeta\left(x_{2}\right)\right) \partial_{2} \sigma_{12} \\
\partial_{1} \sigma_{12}+d\left(\omega, \zeta\left(x_{2}\right)\right) \partial_{2} \sigma_{22}
\end{array}\right) \\
\varepsilon_{p m l}(u)=\left(\begin{array}{cc}
\partial_{1} u_{1} & \frac{1}{2}\left(\partial_{1} u_{2}+d\left(\omega, \zeta\left(x_{2}\right)\right) \partial_{2} u_{1}\right) \\
\frac{1}{2}\left(\partial_{1} u_{2}+d\left(\omega, \zeta\left(x_{2}\right)\right) \partial_{2} u_{1}\right) & d\left(\omega, \zeta\left(x_{2}\right)\right) \partial_{2} u_{2}
\end{array}\right) .
\end{gathered}
$$

$\mathrm{RR} \mathrm{n}^{\circ} 4304$ 


\subsection{Some instructive numerical simulations}

In this section, we will show some simulations with five different homogeneous media, one is isotropic and the four others are orthotropic. The computational domain is a square surrounded with PMLs (see Fig. 3 - left). The numerical method used to solve the elastodynamic equations is based on a mixed formulation of the equations and is described in [8].
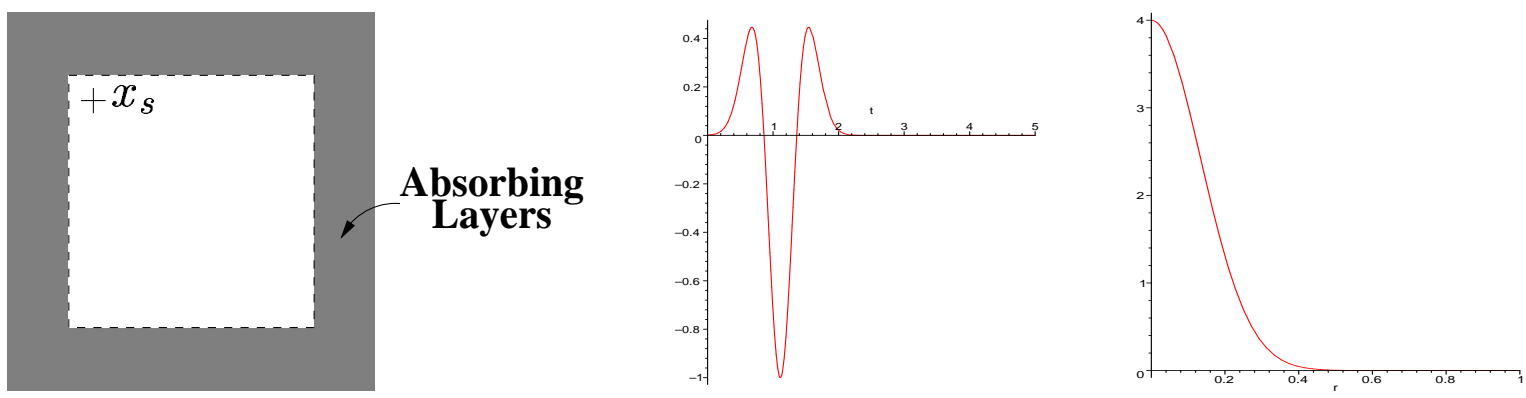

Figure 3: Computational domain (left) - Function $h(t)$ (center) - Function $g(r)$ (right)

In all the simulations, the initial data are taken equal to zero and the source is introduced as a right hand side in equation (3):

$$
f(x, t)=h(t) g\left(\left|x-x_{S}\right|\right) \vec{e}_{1}
$$

where $h$ is the so-called second order Ricker signal with central frequency equal to $f_{0}=0.9 \mathrm{~Hz}$, namely (see also Fig 3):

$$
h(t)=\left[2 \pi^{2}\left(f_{0} t-1\right)^{2}-1\right] e^{-\pi^{2}\left(f_{0} t-1\right)^{2}} .
$$

The function $g(r)$ (see Fig 3) is such that the support of $g\left(\left|x-x_{S}\right|\right.$ ) is concentrated in a small disk centered at the source point $x_{S}$ which is located closed to the absorbing layer (see Fig 3 ) and $\vec{e}_{1}$ denotes the first vector of the canonical basis of $\mathbb{R}^{2}$. We choose the density $\rho=1$ in all experiments.

We represent, for each experiment:

(a) the slowness curves and the wave fronts of the material,

(b) the distribution in space of the norm of the displacement field (snapshots) at several times.

Isotropic Medium. The first simulation is done in an isotropic medium, with Lamé's constants:

$$
\lambda=16, \quad \mu=2 .
$$

The source creates both $\mathrm{P}$ and $\mathrm{S}$ waves (of respective velocities $2 \sqrt{5} \simeq 4.47$ and $\sqrt{2} \simeq 1.414$ ) that correspond to the two successive wavefronts appearing in the snapshots of the solution. One can see that these two waves are perfectly absorbed by the absorbing layer and that, even after a long while $(t=500 s)$, the solution remains equal to zero (see Fig. 5). 

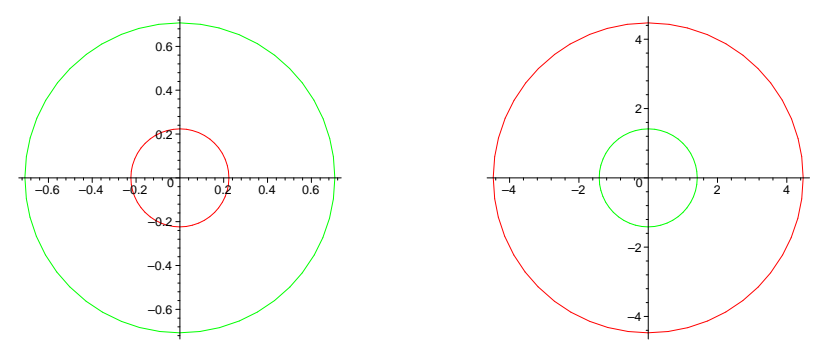

Figure 4: Slowness curves and Wave fronts for the isotropic media.

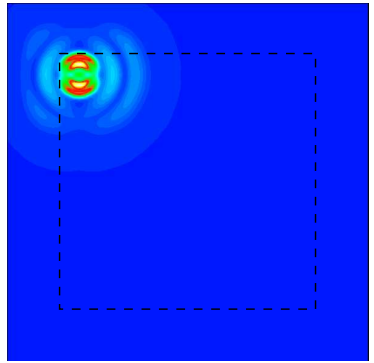

$\mathrm{t}=2 \mathrm{~s}$

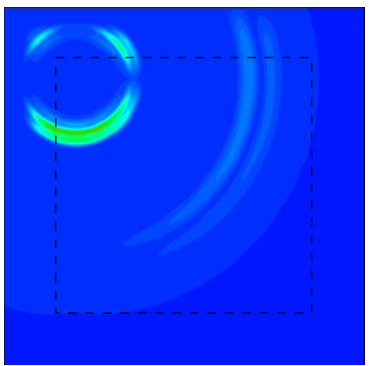

$\mathrm{t}=5 \mathrm{~s}$

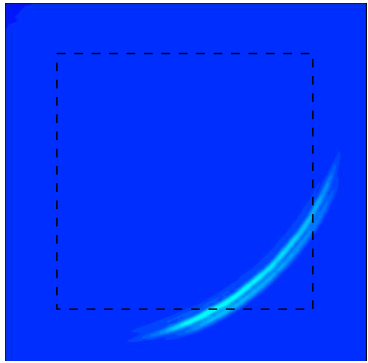

$\mathrm{t}=20 \mathrm{~s}$

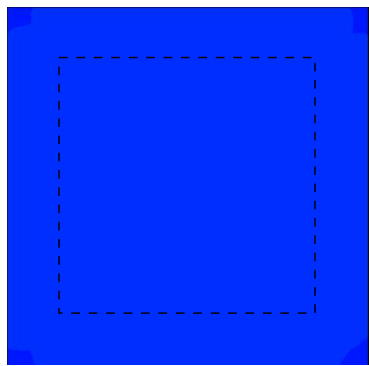

$\mathrm{t}=500 \mathrm{~s}$

Figure 5: Some snapshots at different times for the isotropic media.

Simulation in an anisotropic medium (I). In this example, the elasticity coefficients are given by:

$$
c_{11}=4, c_{22}=20, c_{33}=2 \text { and } c_{12}=3.8 \text {. }
$$

The slowness curves and wave fronts reprensented in Fig. 6 illustrate the anisotropy of the medium. Note that the sets enclosed by the slowness curves remain convex as in the isotropic case. The snapshots of the corresponding numerical experiment are given in Fig. 7.
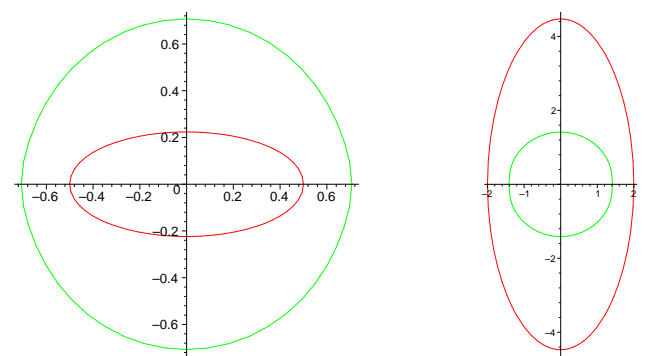

Figure 6: Slowness curves and Wave fronts for for the orthotropic medium (I). 


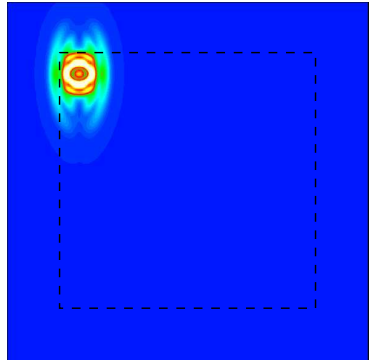

$\mathrm{t}=2 \mathrm{~s}$

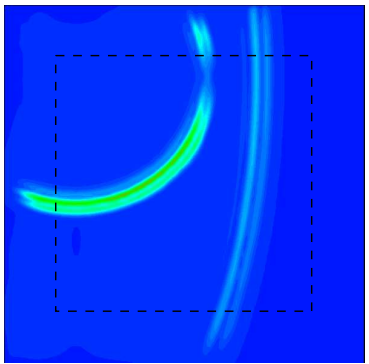

$\mathrm{t}=10 \mathrm{~s}$

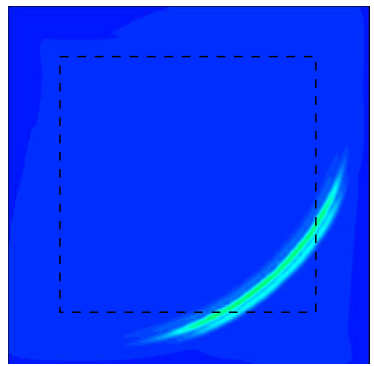

$\mathrm{t}=20 \mathrm{~s}$

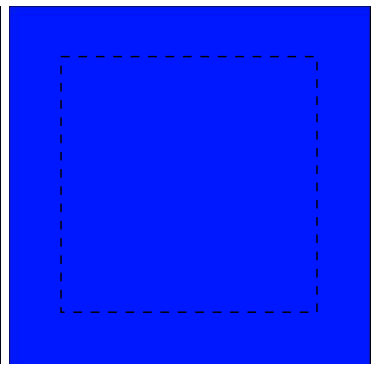

$\mathrm{t}=500 \mathrm{~s}$

Figure 7: Some snapshots at different times for the orthotropic medium (I).

They show that the PML's work pretty well. In particular, they are stable: the solution does not blow up, even after a long time.

Simulation in an anisotropic medium (II). This time, the material is characterized by its elasticity coefficients:

$$
c_{11}=c_{22}=20, \quad c_{33}=2, \quad c_{12}=3.8
$$
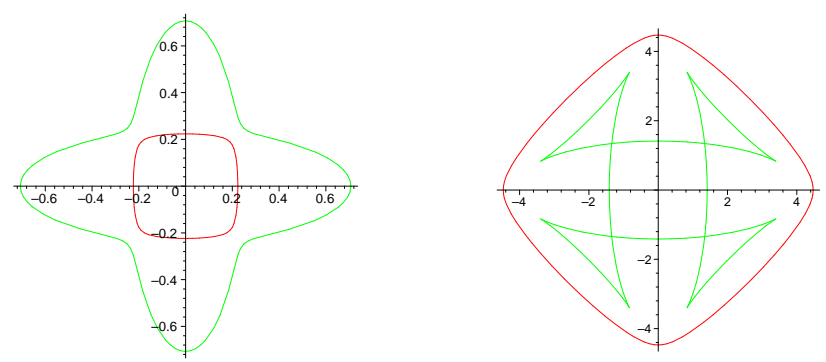

Figure 8: Slowness curves and Wave front for the orthotropic medium (II).

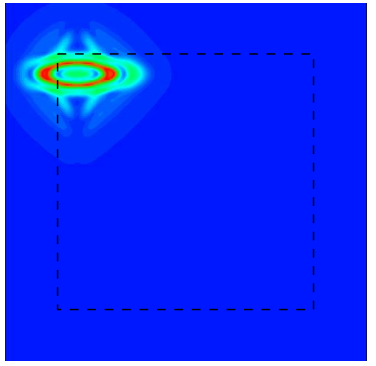

$\mathrm{t}=2 \mathrm{~s}$

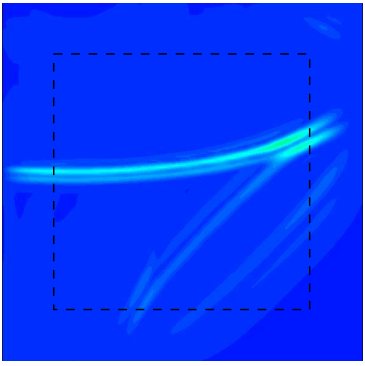

$\mathrm{t}=8 \mathrm{~s}$

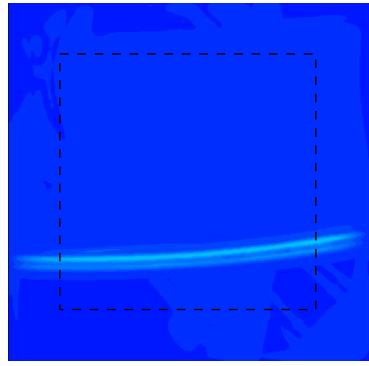

$\mathrm{t}=14 \mathrm{~s}$

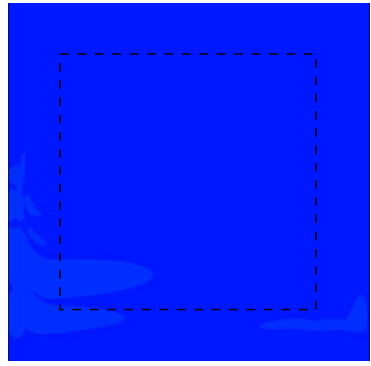

$\mathrm{t}=500 \mathrm{~s}$

Figure 9: Some snapshots at different times for the orthotropic medium (II).

In figure 8 , the medium appears to be much more anisotropic than the previous one. In particular, the set enclosed by the slowness curve of the $Q S$ wave is no longer convex, which gives rise to triplications of the wave front. However, one can see in figure 9 that the PML model still works very well and does not lead to any instability.

Simulation in an anisotropic medium (III). For this experiment, we choose as elasticity coefficients:

$$
c_{11}=4, \quad c_{22}=20, \quad c_{33}=2, \quad c_{12}=4.9
$$



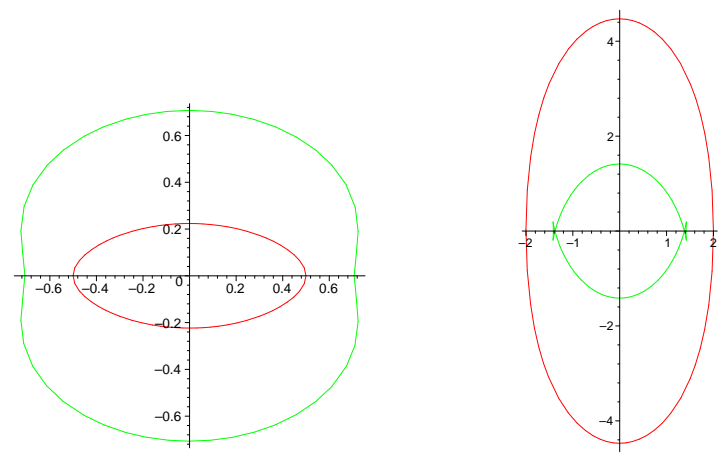

Figure 10: Slowness curves and Wave front for the orthotropic media (III).

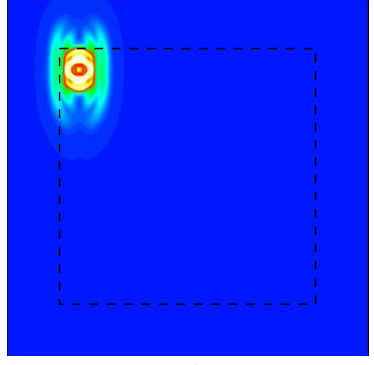

$\mathrm{t}=2 \mathrm{~s}$

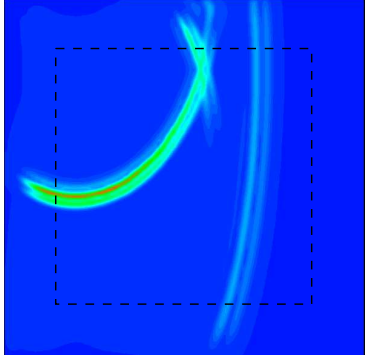

$\mathrm{t}=10 \mathrm{~s}$

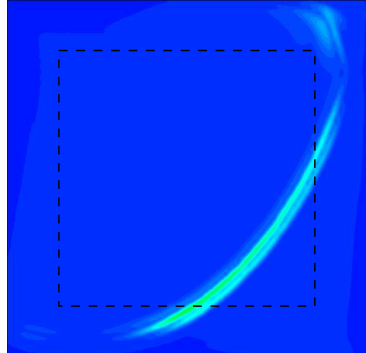

$\mathrm{t}=20 \mathrm{~s}$

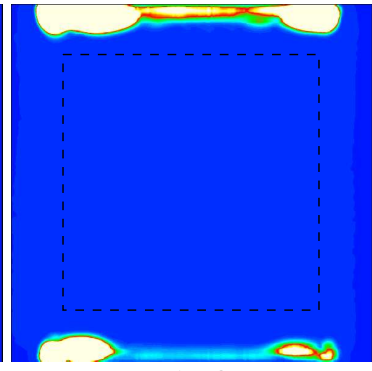

$\mathrm{t}=160 \mathrm{~s}$

Figure 11: Some snapshots at different times for the orthotropic media (III).

Looking at the slowness curves (see Fig. 10), this medium seems to be less anisotropic than the previous one. However, the snapshots of the solutions show that an instability is developing in the PML layer parallel to the $x_{2}$ direction (see Fig. 11 at time $t=160 s$ ). On the contrary, the PML in the $x_{1}$-direction seems to absorb the waves quite well.

Simulation in an anisotropic medium (IV). Our last medium is characterized by the following elasticity coefficients:

$$
c_{11}=4, \quad c_{22}=20, \quad c_{33}=2, \quad c_{12}=7.5
$$

Once again, this is a medium which gives rise to triplications of the QS wave front (see Fig. 12).
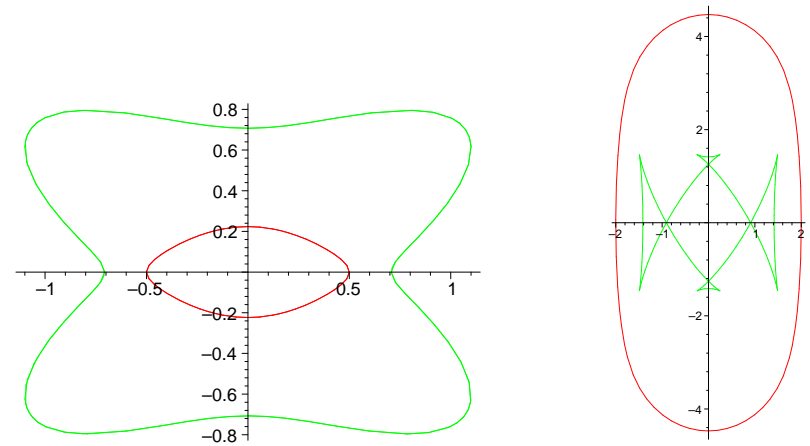

Figure 12: Slowness curves and Wave front for the orthotropic media (IV). 


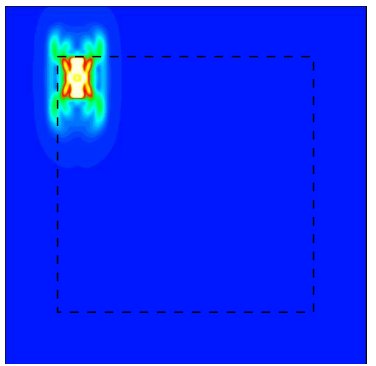

$\mathrm{t}=2 \mathrm{~s}$

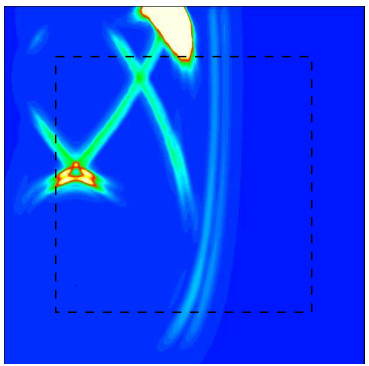

$\mathrm{t}=8 \mathrm{~s}$

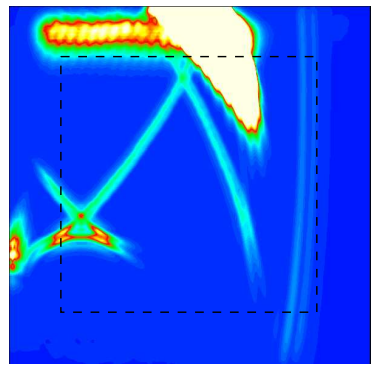

$\mathrm{t}=12 \mathrm{~s}$

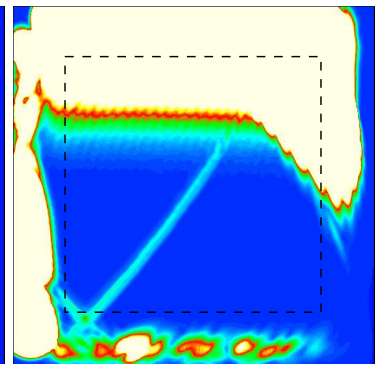

$\mathrm{t}=20 \mathrm{~s}$

Figure 13: Some snapshots at different times for the orthotropic media (IV).

On the snapshots (see Fig. 13), we can see two instabilities appearing very soon in the two PML layers. These instabilities clearly occur when the lowest wave, namely the QS wave, penetrates the absorbing layer.

In conclusion, it seems that the stability of the PML model depends on the physical properties of the media. The aim of this paper is to understand this phenomenon. The explanation of the instabilities observed in the last two simulations, is that these models do not satisfied a very general necessary condition of stability for the PMLs. This necessary condition has a very simple geometrical interpretation that we present in the next section.

\section{A necessary stability condition for PML models associated to gen- eral hyperbolic system. Interpretation in terms of slowness curves.}

In this section, we analyze the PML model (21) (or (24)) for a general hyperbolic system. We restrict ourselves to the case where the absorption coefficient $\zeta$ is constant in space, which makes possible the use of the Fourier analysis. This is necessary step towards the analysis of the non-constant coefficient case. Moreover, there is a general theory for the link between the analysis of the constant coefficient equation and the one of variable coefficient equation. This is the so-called frozen coefficient technique (see [16]).

\subsection{Well-posedness and stability: definition and characterization through plane wave analysis}

We consider the Cauchy problem in the whole space $\mathbb{R}^{2}$ associated to the system:

$$
\left\{\begin{array}{l}
\partial_{t} u^{1}+\zeta u^{1}-A_{1} \partial_{1} u^{1}-A_{1} \partial_{1} u^{2}=0 \\
\partial_{t} u^{2}-A_{2} \partial_{2} u^{1}-A_{2} \partial_{2} u^{2}=0
\end{array}\right.
$$

where $\zeta$ is a strictly positive constant and we set $U=\left(u^{1}, u^{2}\right)$.

Definition 1 We will say that the Cauchy problem (27) associated to the initial data $U_{0}$ is weakly (resp. strongly) well-posed if for any $U(., 0)=U_{0}$ given in the Sobolev space $H^{s}, s>0$ (resp. $s=0$ ), (27) admits a unique solution $U(t)$ that satisfies an estimate on the type

$$
\|U(., t)\|_{L^{2}} \leq K e^{\alpha t}\left\|U_{0}\right\|_{H^{s}}
$$

We will say that the problem is weakly (resp. strongly) stable if it is weakly (or strongly) well-posed and if the solution $U(t)$ satisfies an estimate on the type

$$
\|U(., t)\|_{L^{2}} \leq K(1+t)^{s}\left\|U_{0}\right\|_{H^{s}}
$$


with $s>0$ (resp. $s=0)$.

In what follows we will say that the system is stable if it is at least weakly stable. Roughly speaking the difference between a stable system and a well-posed system is that in the first case, exponentially growing solutions are not permitted.

It is well known that the Fourier analysis permits to relate the notions of well-posedness and stability in terms of analysis of plane waves, i.e. solutions of the form

$$
U(x, t)=e^{i(\omega t-k \cdot x)} D, \quad k \in \mathbb{R}^{2}, \quad D \in \mathbb{R}^{2 m}, \quad \omega \in \mathbb{C} .
$$

To study such solutions it is useful to refer to the initial hyperbolic system (20)

$$
\partial_{t} u-A_{1} \partial_{1} u-A_{2} \partial_{2} u=0,
$$

that admits plane wave solutions of the form

$$
u(x, t)=e^{i(\omega t-k \cdot x)} d, \quad k \in \mathbb{R}^{2}, \quad d \in \mathbb{R}^{m}, \quad \omega \in \mathbb{C},
$$

if and only if $k$ and $\omega$ are related by the dispersion relation

$$
F(\omega, k)=0,
$$

where

$$
F(\omega, k)=\operatorname{det}\left(\omega I-k_{1} A_{1}-k_{2} A_{2}\right),
$$

is a homogeneous polynomial in $\omega$ and $k$ of degree $m$. If we consider (31) as an equation in $\omega$, a consequence of the hyperbolicity of the system (20) is that the solution of (31) are real:

$$
\omega=\omega_{j}(k), \quad j=1, \ldots, m, \quad \text { the eigenvalues of } \mathcal{A}(k) .
$$

Moreover, the functions $\omega_{j}(k)$ are homogeneous functions of degree 1 and differentiable with respect to $k$ as soon as the system (20) is strictly hyperbolic.

As for elastic waves, one can define for each mode $\omega(k)=\omega_{j}(k)$, the phase velocity

$$
\mathcal{V}(K)=\frac{\omega(k)}{|k|} \equiv \omega(K)
$$

where $K=k /|k|$, the slowness vector

$$
\overrightarrow{\mathcal{S}}(K)=\frac{K}{\mathcal{V}(K)}=\frac{k}{\omega(k)}
$$

and the group velocity

$$
V_{g}(k)=V_{g}(K)=\nabla_{k} \omega(k)=-\left(\frac{\partial F}{\partial \omega}(\omega, k)\right)^{-1} \nabla_{k} F(\omega, k)
$$

which is orthogonal to the slowness curves, defined as in section 2 . We shall denote by $\left(V_{g}^{1}(k), V_{g}^{2}(k)\right)$ the two components of $V_{g}(k)$ and by $\left(S_{1}(k), S_{2}(k)\right)$ the ones of $\overrightarrow{\mathcal{S}}(K)$.

Going back to the Fourier analysis of the PML system (27), we see that (27) has solutions of the form (30) if and only if $\omega$ and $k$ are related by the perturbed dispersion relation:

$$
F_{p m l}(\omega, k, \zeta) \equiv F\left(\omega(\omega-i \zeta), k_{1} \omega, k_{2}(\omega-i \zeta)\right)=0
$$


This is a polynomial equation in $\omega$ of degree $2 m$. Therefore the dispersion relation in the PMLs defines $2 m$ modes, $\omega_{j}(k, \zeta), j=1, \ldots, 2 m$, solutions of this equation. It is natural that the number of modes is the double of the one in the original problem, since we have doubled the number of unknowns by passing from (20) to (21).

We remind how the notions of well-posedness and stability are intimately related to the plane wave analysis.

Well-posedness. The system (27) is strongly ill-posed if there exists some exponentially growing modes, that is:

$$
\Im m \omega(k, \zeta) \rightarrow-\infty, \quad \text { when }|k| \rightarrow+\infty,
$$

otherwise it is at least weakly well-posed (see [16]).

Stability. The system (27) is stable in the sense of definition 1 if and only if

$$
\forall k \in \mathbb{R}^{m} \text {, the solutions } \omega(k, \zeta) \text { satisfy } \Im m \omega(k, \zeta) \geq 0 \text {. }
$$

Actually if $\omega=\omega_{R}+i \omega_{I}$, the plane wave solution can be written as:

$$
u(x, t)=e^{-\omega_{I} t} e^{i\left(\omega_{R} t-k \cdot x\right)} D
$$

The existence of solutions $\omega$ with negative imaginary parts would correspond to plane wave solutions with exponential growth in time. A stable system does not admit such solutions.

\subsection{A general well-posedness result}

We shall assume here that:

Assumption (A). The system (20) is strongly hyperbolic and the eigenvalues of $\mathcal{A}(k)$ are different from 0 for $k \neq 0$.

This is in particular the case of the $4 \times 4$ elastodynamics system (9) (the four eigenvalues are $\pm \gamma_{Q P}(k)$, $\left.\pm \gamma_{Q S}(k)\right)$.

In this case, we note that system (27) appears as a zero-order approximation of the following system:

$$
\left\{\begin{array}{l}
\partial_{t} u^{1}-A_{1} \partial_{1} u^{1}-A_{1} \partial_{1} u^{2}=0 \\
\partial_{t} u^{2}-A_{2} \partial_{2} u^{1}-A_{2} \partial_{2} u^{2}=0
\end{array}\right.
$$

that can be shown to be strongly hyperbolic thanks to assumption (A). Indeed, if $\left\{\omega_{j}(k), 1 \leq j \leq m\right\}$ are the eigenvalues of $\mathcal{A}(k)$ associated to the eigenvectors $w_{j}(k) \in \mathbb{R}^{m}$ that form a basis of $\mathbb{R}^{m}$, then one easily checks that the eigenvalues of the $2 m \times 2 m$ matrix:

$$
\tilde{A}(k)=\left(\begin{array}{cc}
k_{1} A_{1} & k_{1} A_{1} \\
k_{2} A_{2} & k_{2} A_{2}
\end{array}\right)
$$

are :

- $\omega_{j}(k) \neq 0 \quad(1 \leq j \leq m)$ associated to the eigenvector $\left(k_{1} / \omega_{j}(k) A_{1} w_{j}(k), k_{2} / \omega_{j}(k) A_{2} w_{j}(k)\right)^{t} \in$ $\mathbb{R}^{2 m}$ 
- 0 , eigenvalue of multiplicity $\mathrm{m}$, whose eigenspace, given by $\left\{(w,-w), w \in \mathbb{R}^{m}\right\}$ has dimension $\mathrm{m}$.

Therefore, thanks to assumption (A), the multiplicity of each eigenvalue is equal to the dimension of the corresponding eigenspace, which ensures that $\tilde{A}(k)$ is diagonalisable. As a consequence, the general theory (see [16]) says that the system (27) is well-posed.

Remark 1 If the assumption (A) is not satisfied, i.e. if 0 is an eigenvalue of $A(k)$ at least for some $k \neq 0$, proving the well-posedness of (27) is not obvious and requires further analysis which is more intimately related to the structure of the unperturbed system and of the perturbation too. Maxwell's equations provide an example of such a system (see [1, 3, 18]).

\subsection{A high frequency stability analysis}

In this section, in addition to assumption (A) we shall assume that system (20) is strictly hyperbolic, which is in particular the case of the elastodynamics system (9). In order to analyse the stability of (27), we focus on the sign of $\Im m \omega(k, \zeta)$ for large $|k|$ (high frequencies). For this, it will be convenient to introduce the parameter:

$$
\varepsilon=\frac{\zeta}{|k|}
$$

and to rewrite the dispersion relation (36) as:

$$
F_{p m l}(\mathcal{V}, K, \varepsilon) \equiv F\left(\mathcal{V}(\mathcal{V}-i \varepsilon), K_{1} \mathcal{V}, K_{2}(\mathcal{V}-i \varepsilon)\right)=0
$$

where $\mathcal{V}$ is the phase velocity defined in (33) and $K=k /|k|$ the unit wave vector. Note that looking at small $\varepsilon$ is equivalent to looking at small $\zeta$ (small absorption) or large $|k|$ (high frequencies). We then have the:

Lemma 1 For every given $K$, the equation (41) admits $2 m$ complex solutions that we denote by $\left(\mathcal{V}_{j}(K, \varepsilon)\right)_{j=1, \ldots, 2 m}$, that can be numbered in such a way that they are continuous functions with respect to $K$ and $\varepsilon$. Moreover, $\left(\mathcal{V}_{j}(K, \varepsilon)\right)$ is differentiable in the neighborhood of any point $\left(K_{0}, \varepsilon_{0}\right)$ where $\left(\mathcal{V}_{j}\left(K_{0}, \varepsilon_{0}\right)\right)$ is a simple root of $(41)$.

Proof: Using the expression (32) of $F$, it is easy to see that the equation is a polynomial equation in the variable $\mathcal{V}$ of degree $2 m$ and that the coefficient of order $2 m$ in the polynomial function $F_{p m l}(\mathcal{V}, K, \varepsilon)$, equal to 1 , does not vanish. Therefore the solutions $\mathcal{V}_{j}(K, \varepsilon)$ are continuous functions with respect to $\varepsilon$. The last part of the lemma results from the implicit function theorem.

The unperturbed equation. Equation (41) is a perturbation of the equation obtained for $\varepsilon=0$, which can be written as ( $F$ is homogeneous of degree $m$ )

$$
F_{p m l}(\mathcal{V}, K, 0)=\mathcal{V}^{m} F(\mathcal{V}, K)=0
$$

The solutions $\mathcal{V}$ of this equation are

- the $m$ physical modes $\mathcal{V}_{j}(K, 0)=\omega_{j}(K), \quad j=1, \ldots, m$,

- one mode of order $m, \mathcal{V}_{j}(K, 0)=0, \quad j=m+1, \ldots, 2 m$.

For the general PML model, the stability condition (38) requires in particular that the high frequency solutions, i.e. $\omega(k, \zeta)$ for large $|k|$, have a positive imaginary part. This is what we call a high frequency necessary condition of stability:

RR $n^{\circ} 4304$ 
Definition 2 The system (27) is said to be stable at high frequency if and only if, there exists $\varepsilon_{0}>0$ such that:

$$
\forall \varepsilon<\varepsilon_{0}, \quad \forall K /|K|=1, \quad \Im m \mathcal{V}_{j}(K, \varepsilon) \leq 0, \quad j=1, \ldots, 2 m
$$

We obtain in particular a necessary stability condition by writing that (43) holds for all physical modes $(j=1, \ldots, m)$. This allows us to prove the:

Theorem 1 Let us suppose that system (20) is strictly hyperbolic and that assumption (A) holds. A necessary condition of stability (high frequency stability) of the PML model in the $x_{1}$ direction (27) is that, for all physical modes of the unperturbed system (20):

$$
\forall K=\left(K_{1}, K_{2}\right) /|K|=1, \quad S_{1}(K) \cdot\left(V_{g}^{1}\right)_{1} \geq 0 .
$$

Proof: From the strict hyperbolicity of (20) and assumption (A), we know that for small $\varepsilon$, in the vicinity of physical modes $\mathcal{V}(K)=\mathcal{V}_{j}(K), j=1, \ldots, m$, the function $\varepsilon \rightarrow \mathcal{V}(K, \varepsilon)=\mathcal{V}_{j}(K, \varepsilon)$ has an expansion of the form:

$$
\mathcal{V}(K, \varepsilon)=\mathcal{V}(K)+\alpha(K) \varepsilon+O\left(\varepsilon^{2}\right)
$$

or equivalently

$$
\omega(k, \zeta)=\omega(k)+\alpha(K) \zeta+O(1 /|k|) .
$$

Expanding the equality

$$
F\left(\mathcal{V}(K, \varepsilon), K_{1}\left(1-i \frac{\varepsilon}{\mathcal{V}(K, \varepsilon)}\right)^{-1}, K_{2}\right)=0
$$

in powers of $\varepsilon$, using (45), we get

$$
F(\mathcal{V}(K), K)+\varepsilon \alpha(K) \frac{\partial F}{\partial K_{1}}(\mathcal{V}(K), K)+i \varepsilon \frac{K_{1}}{\mathcal{V}(K)} \frac{\partial F}{\partial \omega}(\mathcal{V}(K), K)+O\left(\varepsilon^{2}\right)=0,
$$

By definition of $\mathcal{V}(K)$ one has $F(\mathcal{V}(K), K)=0$. Then, writing that the term in $\varepsilon$ is equal to 0 , we obtain

$$
\alpha(K)=-i\left(\frac{\partial F}{\partial \omega}(\mathcal{V}(K), K)\right)^{-1}\left(\frac{K_{1}}{\mathcal{V}(K)} \frac{\partial F}{\partial K_{1}}(\mathcal{V}(K), K)\right)
$$

where we have used assumption (A) which says that $\mathcal{V}(K) \neq 0$ and the strict hyperbolicity of (20) which implies that

$$
\frac{\partial F}{\partial \omega}(\mathcal{V}(K), K) \neq 0
$$

Using formulas (35) and (34), we get:

$$
\alpha(K)=i S_{1}(K) V_{g}^{1}(K) .
$$

It is then easy to conclude since (45) shows that for small $\varepsilon$, the sign of $\Im m \mathcal{V}(K, \varepsilon)$ is given by the sign of $\Im m \alpha(K)$. A standard compactness argument is used to prove the existence of $\varepsilon_{0}$.

Geometrical interpretation. The condition (44) expresses the fact that, along the slowness curves, the slowness vector and the group velocity are oriented in the same way with respect to the $\mathrm{Ox}_{2}$ axis. 


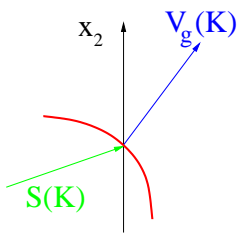

Stable

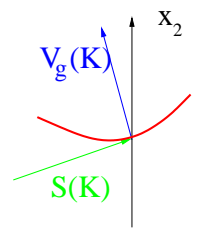

Not stable

Figure 14: Two different configurations. Left: the slowness vector $\vec{S}$ and the group velocity $V_{g}$ are oriented in the same way with respect to the $O x_{2}$ axis. Right: $\vec{S}$ and $V_{g}$ are not oriented in in the same way with respect to the $O x_{2}$ axis.

Obviously, when considering a PML model in the $x_{2}$ direction (i.e. parallel to the $x_{1}$ axis), one has the

Corollary 1 A necessary condition of stability (high frequency stability) of the PML model in the $x_{2}$ direction is that, for all physical modes of the unperturbed system (20):

$$
\forall K=\left(K_{1}, K_{2}\right) /|K|=1, \quad S_{2}(K) \cdot V_{g}^{2}(K) \geq 0
$$

\subsection{Application of the geometrical stability criterion}

\subsubsection{Explanation of the observations of section 2.5 via the analysis of slowness diagrams.}

The geometrical stability condition (44) is clearly satisfied in an isotropic medium. In this case, the slowness curves are composed of two circles and obviously the vectors $\vec{S}$ and $V_{g}$ are parallel. More generally, it is easy to see that (44) will be satisfied as soon as the slowness curves are the boundary of convex sets, which is the case for the orthotropic material (I) considered in section 2.5.

Concerning the three orthotropic materials (II), (III) and (IV) of section 2.5, we have represented in Figure 15 their slowness diagrams. The first remark is that for all the slowness curves associated to the $Q P$ waves (i.e. the inside curve), the geometrical condition is satisfied, since these curves are all convex. This is a general phenomenon that we will prove in the next section (lemma 3). Therefore, the violation of the high frequency stability condition can only come from the $Q S$ waves.

- For material (I), one can see that for all $K$, one has $V_{1}(K) S_{1}(K) \geq 0$ and $V_{2}(K) S_{2}(K) \geq 0$, therefore the high frequency stability condition is satisfied for a layer in the $x_{1}$ direction as well as for a layer in the $x_{2}$ direction. In this case, we did not observe any instability in the numerical results.

- For material (II): one can see that for all $K$, one has $V_{1}(K) S_{1}(K) \geq 0$ which means that the geometrical condition is satisfied for a layer in the $x_{1}$ direction, but there are some vectors $K$ (whose extremities describe the line of medium thickness on the figure) for which $V_{2}(K) S_{2}(K)<$ 0 , therefore the geometrical condition is not satisfied for a layer in the $x_{2}$ direction. This confirms the numerical results: we observed an instability in the PML in $x_{2}$ and not in $x_{1}$.

- For material (III): there are some vectors $K$ (whose extremities describe the thickest line on the figure) for which $V_{1}(K) S_{1}(K)<0$ and there are some other vectors $K$ (whose extremities describe the line of medium thickness on the figure) for which $V_{2}(K) S_{2}(K)<0$ which means that the geometrical condition is not satisfied neither in $x_{1}$ nor in $x_{2}$. This also confirms the numerical results, since we observed instabilities in both layers. 

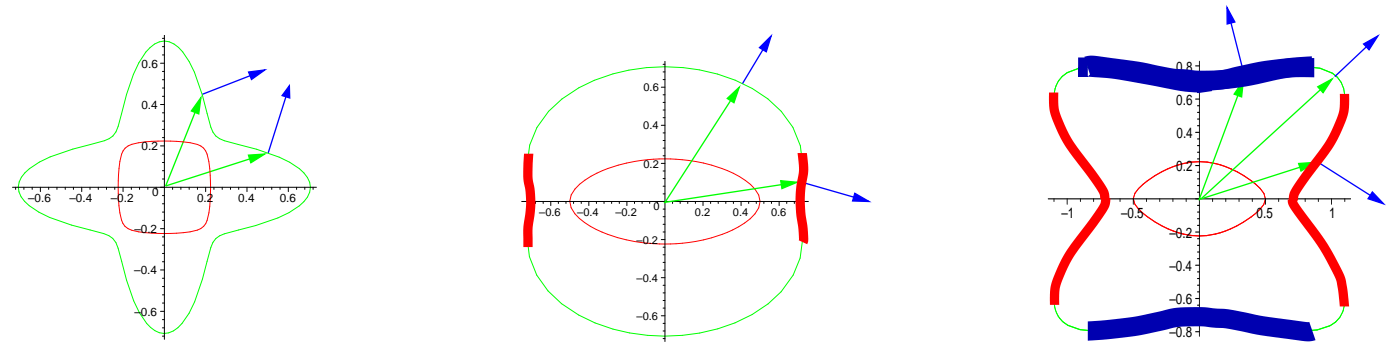

Figure 15: Slowness diagrams. Left: material (II) - Center: material (III) - Right: material (III).

\subsubsection{Application to other mathematical models}

In this section, we show how our geometrical criterion can be applied to show the instability of the PML model applied to other wave phenomena.

\section{The PML model for anisotropic electromagnetic waves.}

Anisotropic dielectric media are characterized by the fact that the electric permittivity $\varepsilon$ is a tensor which is not necessarily proportional to the identity. In $2 \mathrm{D}$, the propagation of electromagnetic waves in such a medium can be describes through the solution $u(x, t)$ of an anisotropic wave equation of the form:

$$
\frac{\partial^{2} u}{\partial t^{2}}-\operatorname{div}(A \nabla u)=0
$$

where $A$ is a $2 \times 2$ symmetric positive definite matrix:

$$
A=\left(\begin{array}{ll}
a & b \\
b & c
\end{array}\right), \quad a>0, \quad c>0, \quad a c-b^{2}>0 .
$$

It appears that the PML model associated to this equation is unstable as soon as the eigenvectors of the matrix $A$ are not parallel to the coordinate axes, i.e. as soon as $b \neq 0$. Indeed, the dispersion relation can be written:

$$
\omega^{2}=A k \cdot k
$$

from which one deduces that the corresponding slowness diagramm is an ellipse whose axes are not parallel to the $x_{1}$ and $x_{2}$ axes. Figure 16 illustrates the fact that the geometrical criterion (44) is not satisfied (this is moreover true for both $x_{1}$ and $x_{2}$ PML layers).

Figure 17 gives an illustration of such an instability : we consider an unbounded anisotropic domain modeled by a square surrounded by PML. $A$ is given by :

$$
a=\frac{3}{4}, b=\frac{3}{2}, c=4
$$

The numerical method is quite the same as in the elastic case : we use a mixed formulation of the equation [8] and consider an explosive source at the middle of the square :

$$
f(x, t)=h(t) g\left(\left|x-x_{S}\right|\right)
$$

where $g$ and $h$ have already been defined in section 2.5. The central frequency is equal to $1 \mathrm{~Hz}$. 


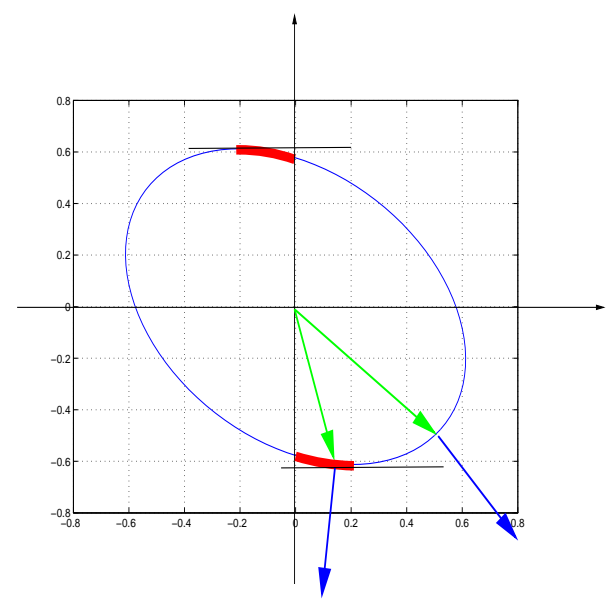

Figure 16: The slowness curve for the anisotropic wave equation.

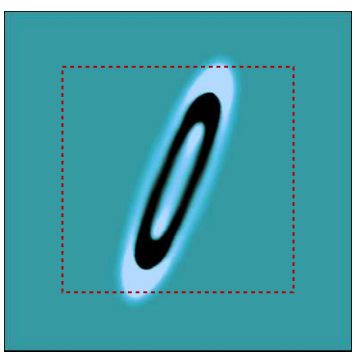

$\mathbf{t}=\mathbf{3} \mathbf{s}$

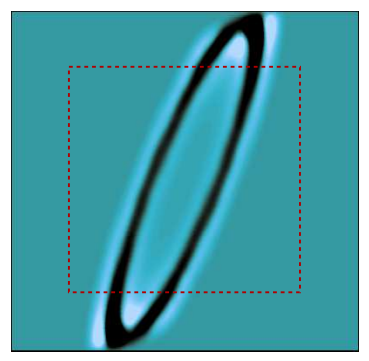

$t=5 \mathrm{~s}$

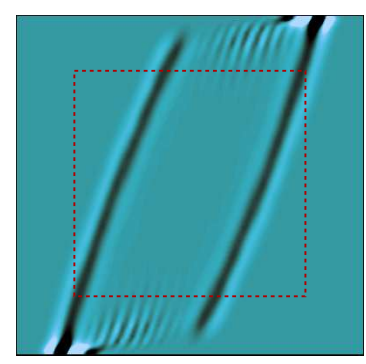

$\mathbf{t}=9 \mathrm{~s}$

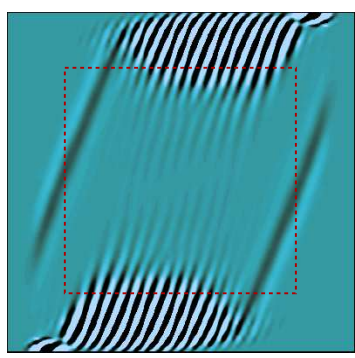

$t=14 \mathrm{~s}$

Figure 17: Instability in the anisotopic acoustic case $\left(a=\frac{3}{4}, b=\frac{3}{2}, c=4\right)$. 


\section{The linearized Euler equations.}

We consider a fluid in which the sound speed is equal to 1 and look at the particular case of a uniform subsonic flow in the direction $x_{1}$ with Mach number $0 \leq M<1$. The linearisation of Euler equations in the neighborhood of such a flow leads to the following advective wave equation which governs the acoustic propagation in such a situation ( $p$ denotes the pressure and $v=\left(v_{1}, v_{2}\right)$ is the velocity field):

$$
\left\{\begin{array}{l}
\frac{\partial p}{\partial t}+M \frac{\partial p}{\partial x_{1}}-\frac{\partial v_{1}}{\partial x_{1}}-\frac{\partial v_{2}}{\partial x_{2}}=0 \\
\frac{\partial v_{1}}{\partial t}+M \frac{\partial v_{1}}{\partial x_{1}}-\frac{\partial p}{\partial x_{1}}=0 \\
\frac{\partial v_{2}}{\partial t}+M \frac{\partial v_{2}}{\partial x_{1}}-\frac{\partial p}{\partial x_{2}}=0
\end{array}\right.
$$

The high frequency geometrical criterium (44) allows us to identify a well known result, namely the instability of the PMLs in the $x_{1}$ direction (e.g., $\left.[14,21,15]\right)$. Indeed, the dispersion relation of (48)

$$
\left(\omega+M k_{1}\right)^{2}-k_{1}^{2}-k_{2}^{2}=0,
$$

defines one slowness curve which is an ellipse with axes parallel to the coordinates axes but not centered at the origin : the center is $\left(M /\left(1-M^{2}\right), 0\right)$. As a consequence, one can see in figure 18 that there are some vectors $K$ (corresponding to the part of the curve of medium thickness) for which $S_{1}(K) V_{1}(K)<0$.

Remark 2 One can however check that the necessary condition for the stability of the PMLs in the $x_{2}$ direction is satisfied. The complete calculations shows that the corresponding system is stable.

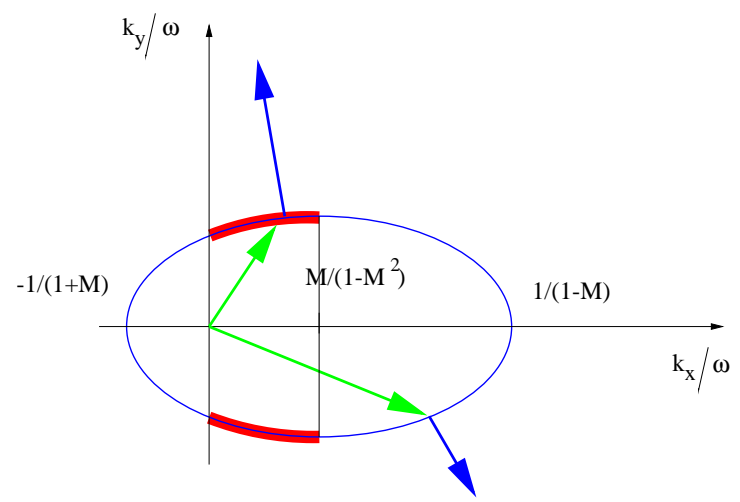

Figure 18: The slowness curve for system (48).

\subsubsection{Other instability phenomena via numerical simulations}

In this section, we present two numerical simulations that seem to indicate that the necessary stability condition (44) is not sufficient for the elastodynamics system. We denote by (V)-1 and (V)-2, these experiments which are done in the same material $(\mathrm{V})$, characterized by the following elasticity coefficients:

$$
c_{11}=10, \quad c_{22}=20, \quad c_{33}=6, \quad c_{12}=2.5
$$


In figure 19, we represent the slowness diagrams (left) and the wave fronts (right). It is clear that the high frequency conditions (44) and (46) are satisfied, for both $x_{1}$ and $x_{2}$ layers since the slowness curves are convex.
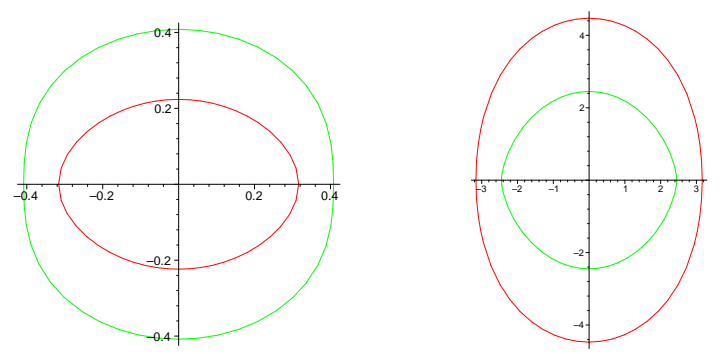

Figure 19: Slowness curves and Wave fronts in the medium (V).

In the experiment (V)-1, we consider a domain with a PML in the $x_{1}$ direction, with reflecting boundary at the top and the bottom of the domain (see figure 20). In the beginning the layers seem to absorb very well the waves. But after a very long while, an instability is developing in the PML layer, as we can see in figure 21.

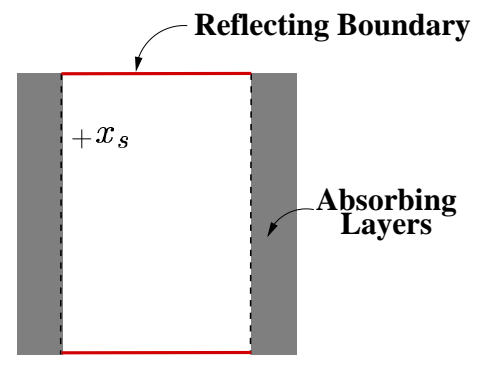

Figure 20: Computational domain for the simulation (V)-1 with $x_{1}$ absorbing layer.

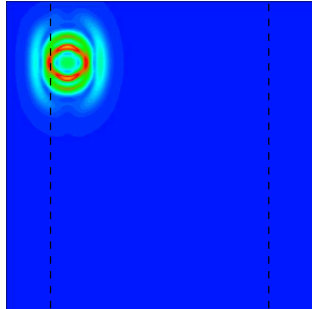

$\mathrm{t}=2 \mathrm{~s}$

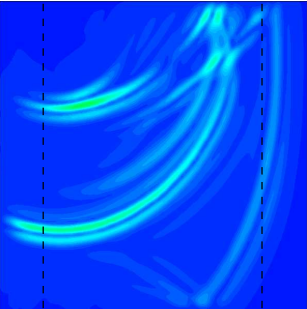

$\mathrm{t}=9 \mathrm{~s}$

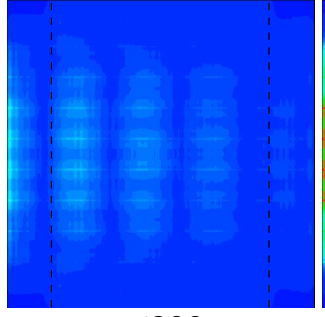

$\mathrm{t}=6800 \mathrm{~s}$

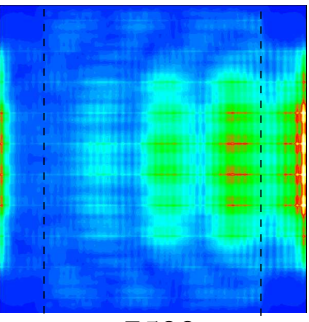

$\mathrm{t}=7500 \mathrm{~s}$

Figure 21: Experiment (V)-1. Some snapshots at different times for the orthotropic medium (V) (with $x_{1}$ absorbing layer).

In the experiment $(\mathrm{V})-2$, the domain of computation as well as the source term is the same as in section 2.5. In figure 22, we represent the snapshots. Again, in the beginning, and during a quite long time, the layers seem to absorb very well the waves. But, after a while, shorter than in the previous experiment, there is an instability appearing in the layers in the $x_{2}$ direction. 


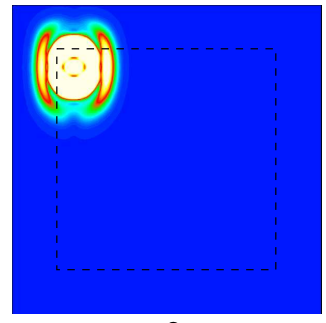

$\mathrm{t}=2 \mathrm{~s}$

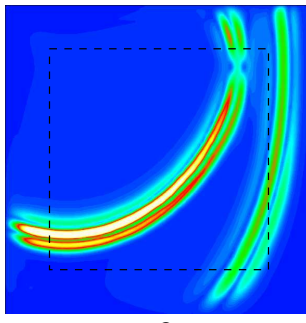

$\mathrm{t}=9 \mathrm{~s}$

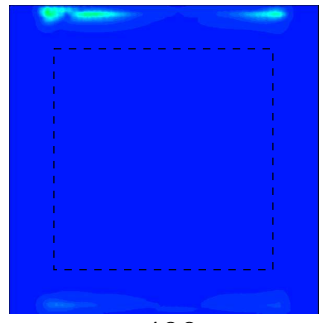

$\mathrm{t}=400 \mathrm{~s}$

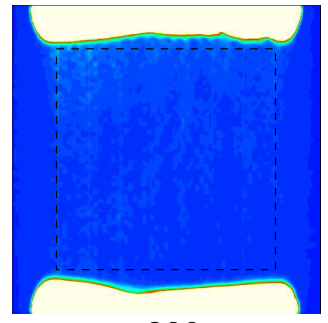

$\mathrm{t}=800 \mathrm{~s}$

Figure 22: Experiment (V)-2. Some snapshots at different times for the orthotropic medium (V).

This suggests additional analysis. We will establish in the next section:

- another necessary stability condition (Lemma 4 and Theorem 3),

- a sufficient stability condition (Theorems 4 and 5).

\section{Stability analysis for the orthotropic elastic model}

We can now go back to the elastodynamic problem (1) and we consider the PML model in the whole space with a constant damping coefficient $\zeta>0$. In this case $m=4$ and the function $F_{p m l}$ appearing in the dispersion relation (42) is defined by:

$$
F_{p m l}(\omega, k, \zeta)=\operatorname{det}\left(\Gamma\left(\omega k_{1},(\omega-i \zeta) k_{2}\right)-\rho \omega^{2}(\omega-i \zeta)^{2} I\right)
$$

In section 4.1, we will show how the high frequency necessary stability condition (44) (coming from the high frequency analysis in the vicinity of the four physical modes) can be expressed in terms of inequalities on the elasticity coefficients. In section 4.2 , we establish a second high frequency necessary stability condition, which comes from the analysis of the solutions in the vicinity of the multiple mode 0 of order $m=4$.

The high frequency stability condition means that, for large $k$, the solutions $\omega(k, \zeta)$ are in the good side of the complex plane (i.e. with a positive imaginary part), but there is no guaranty that they stay in the good side for every frequencies. In section 4.3 , we obtain a condition which implies that the solutions never cross the real axis. Using a continuity argument, this allows to conclude that, under this condition, if the solutions are in the good side of the complex plane at high frequency, they necessarily stay in the good side for all frequencies. In this case, we get a sufficient stability condition. As in section 3 , we introduce the parameter $\varepsilon=\zeta /|k|$ and for convenience, we shall adopt a new notation by introducing the angle $\theta \in[-\pi, \pi$ [ such that

$$
K=\frac{k}{|k|}=(\cos \theta, \sin \theta) \text {. }
$$

Therefore, if we set:

$$
\left(\mathcal{V}_{\theta}\right)_{j}(\varepsilon)=\mathcal{V}_{j}(K, \varepsilon), \quad j=1, \ldots, 8
$$

the functions $\left(\mathcal{V}_{\theta}\right)_{j}(\varepsilon)$ are the 8 branches of solutions of the dispersion relation, considered as an equation in the phase velocity $\mathcal{V}(11)$ :

$$
F_{p m l}(\mathcal{V}, \theta, \varepsilon) \equiv F_{p m l}(\mathcal{V}, K, \varepsilon)=0
$$

where $F_{p m l}$ has been defined in (50). We shall assume that the functions $\left(\mathcal{V}_{\theta}\right)_{j}(\varepsilon)$ are numbered in such a way that 
- $\left(\left(\mathcal{V}_{\theta}\right)_{j}(\varepsilon)\right)_{j=1,2}$, the two branches issued from the physical modes QP:

$$
\left(\mathcal{V}_{\theta}\right)_{j}(0)=\mathcal{V}_{j}(K, 0)= \pm \omega_{Q P}(K), \quad j=1,2,
$$

- $\left(\left(\mathcal{V}_{\theta}\right)_{j}(\varepsilon)\right)_{j=3,4}$, the two branches issued from the physical modes QS:

$$
\left(\mathcal{V}_{\theta}\right)_{j}(0)=\mathcal{V}_{j}(K, 0)= \pm \omega_{Q S}(K), \quad j=3,4,
$$

- $\left(\left(\mathcal{V}_{\theta}\right)_{j}(\varepsilon)\right)_{j=5, . ., 8}$, the four branches issued from the multiple root of order 4

$$
\left(\mathcal{V}_{\theta}\right)_{j}(0)=\mathcal{V}_{j}(K, 0)=0, \quad j=5, \ldots, 8
$$

It is easy to see that:

$$
\forall \theta \in\left[-\pi, \pi\left[, \quad F_{p m l}(\mathcal{V}, \theta, \varepsilon)=F_{p m l}(\mathcal{V},-\theta, \varepsilon)=F_{p m l}(\mathcal{V}, \pi-\theta, \varepsilon)\right.\right.
$$

As a consequence, if

$$
S(\theta, \varepsilon)=\left\{\left(\mathcal{V}_{\theta}\right)_{j}(\varepsilon), j=1, \ldots, 8\right\},
$$

denotes the set of solutions of (51), one has:

$$
\forall \theta \in[-\pi, \pi[, \forall j=1, \ldots, 8, \quad S(\theta, \varepsilon)=S(-\theta, \varepsilon)=S(\pi-\theta, \varepsilon)
$$

which justifies in what follows to restrict ourselves to:

$$
\theta \in[0, \pi / 2] .
$$

The two limit values $\theta=0$ and $\theta=\pi / 2$ do not pose any problem as shown is the following (whose proof is immediate):

Lemma 2 For $\theta=0$, the solutions of (51) are given by:

$$
\left\{\begin{array}{l}
\left(\mathcal{V}_{0}(\varepsilon)\right)_{1,2} \equiv\left(\mathcal{V}_{0}(\varepsilon)\right)_{Q P}^{ \pm}= \pm \sqrt{\max \left(c_{11}, c_{33}\right)}+i \varepsilon, \text { of order } 1 \\
\left(\mathcal{V}_{0}(\varepsilon)\right)_{3,4} \equiv\left(\mathcal{V}_{0}(\varepsilon)\right)_{Q S}^{ \pm}= \pm \sqrt{\min \left(c_{11}, c_{33}\right)}+i \varepsilon, \quad \text { of order } 1 \\
\left(\left(\mathcal{V}_{0}(\varepsilon)\right)_{j}\right)_{j=5, . ., 8}=0, \quad \text { of order } 4
\end{array}\right.
$$

For $\theta=\pi / 2$, the solutions of (51) are given by :

$$
\left\{\begin{array}{l}
\left(\mathcal{V}_{\pi / 2}(\varepsilon)\right)_{1,2} \equiv\left(\mathcal{V}_{\pi / 2}(\varepsilon)\right)_{Q P}^{ \pm}= \pm \sqrt{\max \left(c_{22}, c_{33}\right)}, \quad \text { of order } 1 \\
\left(\mathcal{V}_{\pi / 2}(\varepsilon)\right)_{3,4} \equiv\left(\mathcal{V}_{\pi / 2}(\varepsilon)\right)_{Q S}^{ \pm}= \pm \sqrt{\min \left(c_{22}, c_{33}\right)}, \quad \text { of order } 1 \\
\left(\left(\mathcal{V}_{\pi / 2}(\varepsilon)\right)_{j}\right)_{j=5, . ., 8}=i \varepsilon, \quad \text { of order } 4 .
\end{array}\right.
$$

In particular $\Im m\left(\mathcal{V}_{\pi / 2}(\varepsilon)\right)_{j} \geq 0$ and $\Im m\left(\mathcal{V}_{0}(\varepsilon)\right)_{j} \geq 0, j=1, . ., 8$.

It remains to consider $\theta \in] 0, \pi / 2[$. 


\subsection{A first high frequency necessary stability condition}

We have established in Theorem 1 the high frequency necessary stability condition (44), which expresses the fact that, in the vicinity of the physical modes (i.e. for small $\varepsilon$ ), the branches of the solutions $\left(\left(\mathcal{V}_{\theta}\right)_{j}(\varepsilon)\right)_{j=1 . .4}$ are in the good side of the complex plane (i.e. with a positive imaginary part). We give here an equivalent form of condition (44) expressed in terms of the elasticity coefficients.

Theorem 2 The necessary condition of stability (44) is equivalent to:

$$
\left\{\left(c_{12}+c_{33}\right)^{2}-c_{11}\left(c_{22}-c_{33}\right)\right\} \times\left\{\left(c_{12}+c_{33}\right)^{2}+c_{33}\left(c_{22}-c_{33}\right)\right\} \leq 0 .
$$

Proof. In order to prove (44), we have to show that, for all physical modes, the quantity:

$$
S_{1}(K) \cdot V_{g}^{1}(K)=\frac{K_{1}}{\mathcal{V}(K)} \partial_{k_{1}} \mathcal{V}(K)=\frac{K_{1}}{2 \mathcal{V}(K)^{2}} \partial_{k_{1}}\left(\mathcal{V}(K)^{2}\right)
$$

is positive for all unit vectors $K$. As $\mathcal{V}(K)$ is characterized by the fact that $\gamma(K)=\rho \mathcal{V}(K)^{2}$ is one of the eigenvalues of Chrystoffel's tensor $\Gamma(K)(16)$, we have to study the sign of:

$$
Q(\theta)=K_{1} \times \frac{\partial \gamma}{\partial k_{1}}(K)
$$

for all $K=(\cos \theta, \sin \theta)$, and for the two positive eigenvalues of $\Gamma(K)$ :

$$
\gamma(K)=\gamma_{Q P}(K)=\frac{1}{2}(T+\sqrt{\Delta}) \text { and } \gamma(K)=\gamma_{Q S}(K)=\frac{1}{2}(T-\sqrt{\Delta})
$$

where:

$$
\left\{\begin{array}{l}
T=\left(c_{11}+c_{33}\right) K_{1}^{2}+\left(c_{33}+c_{22}\right) K_{2}^{2} \\
\Delta=\left[\left(c_{11}-c_{33}\right) K_{1}^{2}+\left(c_{33}-c_{22}\right) K_{2}^{2}\right]^{2}+4 K_{1}^{2} K_{2}^{2}\left(c_{12}+c_{33}\right)^{2}
\end{array}\right.
$$

Therefore (44) is satisfied if and only if $Q^{+}(\theta) \geq 0$ and $Q^{-}(\theta) \geq 0$ for all $\theta$, with

$$
Q^{ \pm}(\theta)=\frac{1}{2} K_{1}\left(\frac{\partial T}{\partial K_{1}} \pm \frac{1}{2} \frac{\partial \Delta}{\partial K_{1}} \frac{1}{\sqrt{\Delta}}\right)=\frac{K_{1}^{4}}{\sqrt{\Delta}} Q_{2}^{ \pm}(\theta)
$$

where, if $t=\tan \theta=K_{2} / K_{1}$, one easily computes that:

$$
Q_{2}^{ \pm}(\theta)=a(t) \pm b(t)
$$

with

$$
\left\{\begin{array}{l}
a(t)=\left(c_{11}+c_{33}\right) \sqrt{\alpha(t)} \quad(\geq 0), \\
b(t)=\left(c_{11}-c_{33}\right)^{2}+\left(\alpha_{1}+\alpha_{2}\right) t^{2}, \\
\alpha(t)=\left(\left(c_{11}-c_{33}\right)+\left(c_{33}-c_{22}\right) t^{2}\right)^{2}+4 t^{2}\left(c_{12}+c_{33}\right)^{2},
\end{array}\right.
$$

where the coefficients $\alpha_{1}$ and $\alpha_{2}$ are given by

$$
\left\{\begin{array}{l}
\alpha_{1}=\left(c_{12}+c_{33}\right)^{2}-c_{11}\left(c_{22}-c_{33}\right) \\
\alpha_{2}=\left(c_{12}+c_{33}\right)^{2}+c_{33}\left(c_{22}-c_{33}\right)
\end{array}\right.
$$


Note that, as $a(t)$ is positive, one has the equivalence:

$$
\Longleftrightarrow\left\{\begin{array}{ll}
a(t)+b(t) \geq 0, & \forall t>0, \\
a(t)-b(t) \geq 0, & \forall t>0,
\end{array} \quad \Longleftrightarrow \quad a(t)^{2}-b(t)^{2} \geq 0, \quad \forall t>0 .\right.
$$

One computes that :

$$
a(t)^{2}-b(t)^{2}=4\left(a_{4} t^{4}+2 a_{2} t^{2}+a_{0}\right),
$$

with :

$$
\left\{\begin{array}{l}
a_{4}=-\alpha_{1} \alpha_{2}, \\
a_{2}=c_{11} c_{33}\left(\alpha_{1}+\alpha_{2}\right), \\
a_{0}=c_{11} c_{33}\left(c_{11}-c_{33}\right)^{2} \geq 0 .
\end{array}\right.
$$

Therefore (44) is equivalent to:

$$
\phi(X) \equiv a_{4} X^{2}+2 a_{2} X+a_{0} \geq 0, \quad \forall X \geq 0 .
$$

To conclude, we first observe that condition $\left(\mathcal{C}_{1}\right)$ is nothing but $a_{4} \geq 0$.

Obviously, (60) implies $a_{4} \geq 0$. Conversely, assuming $a_{4} \geq 0$,

- If $a_{2}^{2}<a_{0} a_{4}, \Phi(X)$ has a constant sign. As $\Phi(0)=a_{0} \geq 0$, one has $\Phi(X) \geq 0, \forall X \geq 0$.

- If $a_{2}^{2} \geq a_{0} a_{4}, \Phi(X)$ has two real zeros and condition (60) is equivalent to saying that these two roots are negative. Since $a_{0} a_{4} \geq 0$, the two roots have the same sign. To conclude, it suffishes to observe by a simple computation that:

$$
a_{2}^{2}-a_{0} a_{4}=c_{11} c_{33}\left(c_{12}+c_{33}\right)^{2}\left[a_{2}-\left(c_{12}+c_{33}\right)^{2}\right]
$$

Therefore $a_{2}^{2}-a_{0} a_{4} \geq 0$ implies $a_{2} \geq\left(c_{12}+c_{33}\right)^{2} \geq 0$. As $a_{0} \geq 0$, this means that the sum of the two roots is negative and thus that each of them is negative.

Theorem 2 means that, if $\left(\mathcal{C}_{1}\right)$ is satisfied, then one has for small $\varepsilon$ :

$$
\left.\Im m\left(\left(\mathcal{V}_{\theta}\right)_{j}(\varepsilon)\right) \geq 0, \quad \forall \theta \in\right] 0, \pi / 2[, \quad \forall j=1, \ldots, 4 .
$$

In the following lemma, we make the result more precize: we show that the instability can not come from the vicinity of the $Q P$ waves, which is in some sense a theoretical confirmation of what we have observed in the numerical experiments of section 2 .

Lemma 3 For any orthotropic material, one has the following property, for small $\varepsilon$ :

$$
\left.\Im m\left(\left(\mathcal{V}_{\theta}\right)_{j}\right)(\varepsilon) \geq 0, \quad \forall \theta \in\right] 0, \pi / 2[, \quad j=1,2 .
$$

Proof. In this proof, we use the same notations as in the proof of theorem 2. Let us assume that there is an instability in the vicinity of the QP wave, that means that for $j=1$ or $j=2$, one has :

$$
\exists \theta \in] 0, \pi / 2\left[, \quad \Im m\left(\left(\mathcal{V}_{\theta}^{1}\right)_{j}\right)<0 .\right.
$$

This also means (see proof of theorem 2) that

$$
\exists t>0, \quad a(t)+b(t)<0 .
$$

$\mathrm{RR} \mathrm{n}^{\circ} 4304$ 
From theorem 2, this implies that $\left(\mathcal{C}_{1}\right)$ is not satisfied which is equivalent to say that $a_{4}<0$. Then :

$$
\alpha_{1} \alpha_{2}>0 \text {. }
$$

Since $a(t) \geq 0,(63)$ implies in particular that $b(t)<0$, which is possible only if :

$$
\alpha_{1}+\alpha_{2}<0 \text {. }
$$

The two inequalities (64) and (65) thus implies that $\alpha_{1}<0$ and $\alpha_{2}<0$. So, we have:

$$
\left(c_{12}+c_{33}\right)^{2}<-c_{33}\left(c_{22}-c_{33}\right) \text { and }\left(c_{12}+c_{33}\right)^{2}<c_{11}\left(c_{22}-c_{33}\right) .
$$

Since the two right hand sides are of opposite sign, this would imply that $\left(c_{12}+c_{33}\right)^{2}<0$ which is impossible.

\subsection{A second high frequency necessary stability condition}

We assume in this section that $\theta \in] 0, \pi / 2[$.

Lemma 4 The four solutions $\left(\mathcal{V}_{\theta}(\varepsilon)\right)_{j}, j=5, . ., 8$, satisfy :

$$
\left.\Im m\left(\left(\mathcal{V}_{\theta}^{1}\right)_{j}\right) \geq 0, \quad \forall \theta \in\right] 0, \pi / 2[, \quad \forall j=5, \ldots, 8
$$

if and only if the two following inequalities are satisfied;

$$
\left\{\begin{array}{l}
\left(c_{12}+2 c_{33}\right)^{2} \leq c_{11} c_{22}, \\
\left(c_{12}+c_{33}\right)^{2} \leq c_{11} c_{22}+c_{33}^{2} .
\end{array}\right.
$$

Proof. In what follows, $\mathcal{V}_{\theta}(\varepsilon)$ is one of the functions $\left(\mathcal{V}_{\theta}\right)_{j}(\varepsilon)$ for $j=5, \ldots, 8$. Let us substitute the expansion (we know that such an expansion exists since we have weak well-posedness):

$$
\mathcal{V}_{\theta}(\varepsilon)=\varepsilon \mathcal{V}_{\theta}^{1}+o(\varepsilon)
$$

into the equation :

$$
F_{p m l}\left(\mathcal{V}_{\theta}(\varepsilon), \theta, \varepsilon\right)=0 .
$$

We obtain, by identifying the lower order term in $\varepsilon$ :

$$
\mid \begin{gathered}
{\left[c_{11}\left(\mathcal{V}_{\theta}^{1}\right)^{2} \cos ^{2} \theta+c_{33}\left(\mathcal{V}_{\theta}^{1}-i\right)^{2} \sin ^{2} \theta\right]\left[c_{33}\left(\mathcal{V}_{\theta}^{1}\right)^{2} \cos ^{2} \theta+c_{22}\left(\mathcal{V}_{\theta}^{1}-i\right)^{2} \sin ^{2} \theta\right]} \\
-\left(c_{12}+c_{33}\right)^{2}\left(\mathcal{V}_{\theta}^{1}\right)^{2}\left(\mathcal{V}_{\theta}^{1}-i\right)^{2} \cos ^{2} \theta \sin ^{2} \theta=0 .
\end{gathered}
$$

It is easy to see that for $\theta \in] 0, \pi / 2\left[\right.$, the solution $\mathcal{V}_{\theta}^{1}$ does not vanish, then we can make the change of unknown :

$$
z=\frac{\mathcal{V}_{\theta}^{1}-i}{\mathcal{V}_{\theta}^{1}} \tan \theta
$$

which leads to a polynomial equation in $z$ whose coefficients are independent of $\theta$ :

$$
A_{4} z^{4}+A_{2} z^{2}+A_{0}=0
$$

with

$$
\left\{\begin{array}{l}
A_{4}=c_{22} c_{33}>0, \\
A_{2}=c_{11} c_{22}+c_{33}^{2}-\left(c_{12}+c_{33}\right)^{2}, \\
A_{0}=c_{11} c_{33}>0 .
\end{array}\right.
$$

Let us show the equivalence of the two following conditions: 
(i) The solutions $\mathcal{V}_{\theta}^{1}$ of (67) have a positive imaginary part for all values of $\theta$.

(ii) The solutions $z$ of (69) are purely imaginary.

Indeed, if (ii) holds and if $\left\{i \lambda_{j}, j=5, \ldots, 8\right\}$ are the four roots of (69) then the four solutions of (67) are given by:

$$
\left(\mathcal{V}_{\theta}^{1}\right)_{j}=\frac{i \tan \theta}{\tan \theta-i \lambda_{j}}=\tan \theta \frac{-\lambda_{j}+i \tan \theta}{\tan ^{2} \theta+\lambda_{j}^{2}}
$$

so that:

$$
\Im m\left(\mathcal{V}_{\theta}^{1}\right)_{j}=\frac{\tan ^{2} \theta}{\tan ^{2} \theta+\lambda_{j}^{2}} \geq 0
$$

Conversely, let us assume that $z$ is a solution of (69) with non zero real part (note that $z \neq 0$ ). We can assume that $\Re e(z)>0$ since $-z$ is also a solution. Then, except maybe for $\theta=\theta_{0}$ such that, when $z$ is real, $z=\tan \theta_{0}$, a solution of $(68)$ is given by:

$$
\mathcal{V}_{\theta}^{1}=\frac{i \tan \theta}{\tan \theta-z}=\tan \theta \frac{-\Im m(z)+i(\tan \theta-\Re e(z))}{|\tan \theta-z|^{2}}
$$

with imaginary part:

$$
\Im m \mathcal{V}_{\theta}^{1}=\frac{\tan \theta(\tan \theta-\Re e(z))}{|\tan \theta-z|^{2}}
$$

It suffices to remark that when $\theta$ goes from 0 to $\pi / 2$ the sign of $\tan \theta-\Re e(z)$ changes which means that, for some values of $\theta,(69)$ admits solutions with strictly negative imaginary part.

To conclude, it suffices to remark that (i) is equivalent to saying that the two roots of the polynomial $A_{4} Z^{2}+A_{2} Z+A_{0}$ are real negative, which is equivalent to:

$$
A_{2}^{2}<4 A_{0} A_{4} \quad \text { and } A_{2} \geq 0
$$

what we wanted to show since $c_{11} c_{22}-c_{12}^{2} \geq 0$ and:

$$
A_{2}^{2}-4 A_{0} A_{4}=\left(c_{11} c_{22}-c_{12}^{2}\right)\left[c_{11} c_{22}-\left(c_{12}+2 c_{33}\right)^{2}\right] \text {. }
$$

Theorem 2 and Lemma 4 can be summarized as follows:

Theorem 3 The PML model (26) is stable at high frequency in the sense of definition 2 if and only if conditions $\left(\mathcal{C}_{1}\right)$ and $\left(\mathcal{C}_{2}\right)$ are satisfied.

\subsection{A sufficient stability condition}

From a geometrical point of view, proving the stability of the PML model is equivalent to show that, $\theta$ playing the role of a parameter, the curves in the complex plane described by $\left(\mathcal{V}_{\theta}\right)_{j}(\varepsilon)$ when $\varepsilon$ goes from 0 to $+\infty$ is located in the right side of the complex plane, namely the half-space of complex numbers with positive imaginary part. In the previous sections, we have established conditions $\left(\mathcal{C}_{1}\right)$ and $\left(\mathcal{C}_{2}\right)$ such that it is the case for the first part of the curves corresponding to small $\varepsilon$. In what follows, we are going to establish the conditions for which these curves never meet again the real axis, which will provide us a sufficient stability condition thanks to a continuity argument.

For our purpose, it will be useful to use a new parameterization of the $(\theta, \mathcal{V}, \varepsilon)$ space. More precisely, we consider the transformation (we shall see later that this transformation is one to one):

$$
(\theta, \mathcal{V}, \varepsilon) \in] 0, \frac{\pi}{2}\left[\times \mathbb{R}_{*} \times \mathbb{R}_{*}^{+} \quad \mapsto \quad(X, \tau) \in \mathbb{R}_{*}^{+} \times(\mathbb{C} \backslash \mathbb{R})\right.
$$

$\mathrm{RR} \mathrm{n}^{\circ} 4304$ 
defined by (we set $t=\tan \theta$ ) :

$$
\left\{\begin{array}{c}
X(\theta, \mathcal{V})=\frac{1+t^{2}}{t^{2}} \mathcal{V}^{2} \\
\tau(\theta, \varepsilon, \mathcal{V})=t^{2}\left(\frac{\mathcal{V}-i \varepsilon}{\mathcal{V}}\right)^{2}
\end{array}\right.
$$

By construction, we have:

$$
F_{p m l}(\mathcal{V}, \theta, \varepsilon)=\cos ^{4} \theta \mathcal{V}^{4} G(X(\theta, \mathcal{V}), \tau(\theta, \varepsilon, \mathcal{V}))
$$

with :

$$
G(X, \tau)=\left(c_{11}+\left(c_{33}-X\right) \tau\right)\left(c_{33}+\left(c_{22}-X\right) \tau\right)-\left(c_{12}+c_{33}\right)^{2} \tau .
$$

We shall use this parametrization to express the property which means that the curves $\varepsilon \mapsto \mathcal{V}_{j}(\varepsilon)$ never meet again the real axis, namely

$$
\left.\left(P_{1}\right) \quad \forall \theta \in\right] 0, \pi / 2\left[, \quad \forall j=1, . ., 8, \quad\left(\mathcal{V}_{\theta}(\varepsilon)\right)_{j} \in \mathbb{R} \quad \Longrightarrow \quad \varepsilon=0 .\right.
$$

More precisely, we prove the:

Lemma 5 The property $\left(P_{1}\right)$ is equivalent to:

$$
\left(P_{2}\right) \quad \forall X \in \mathbb{R}^{+}, \quad g(X, \tau)=0 \quad \Longrightarrow \quad \tau \in \mathbb{R} .
$$

Proof. If (P1) does not hold, there exists $\varepsilon_{0}>0$ and $\left.\theta_{0} \in\right] 0, \pi / 2[$ such that, for some $j \in\{1, . ., 8\}$,

$$
\left(\mathcal{V}_{\theta_{0}}\left(\varepsilon_{0}\right)\right)_{j}=\mathcal{V}_{0} \in \mathbb{R}_{*}
$$

If we set $X_{0}=X\left(\theta_{0}, \mathcal{V}_{0}\right) \in \mathbb{R}^{+}$and $\tau_{0}=\tau\left(\theta_{0}, \varepsilon_{0}, \mathcal{V}_{0}\right)$, by definition of $G$ :

$$
F_{p m l}\left(\theta, \varepsilon, \mathcal{V}_{0}\right)=0 \Longrightarrow G\left(X_{0}, \tau_{0}\right)=0 .
$$

As $\mathcal{V}_{0} \in \mathbb{R}_{*}, X_{0}>0$ and $\varepsilon_{0}>0$ implies that $\Im m \tau_{0}=-2 t_{0}^{2} \varepsilon_{0} / \mathcal{V}_{0} \neq 0$, which shows that (P2) does not hold too.

Reciprocally, assume that $\left(P_{2}\right)$ is not satisfied. This means that there exist $\tau_{0} \in C \backslash \mathbb{R}$ and $X_{0}>0$ (it is obvious that $X_{0}=0$ would imply that $\tau_{0} \in \mathbb{R}$ ) such that

$$
G\left(X_{0}, \tau_{0}\right)=0 .
$$

Let us introduce the two real numbers:

$$
t_{0}=\left(\frac{1}{2} \frac{\left(\Im m \tau_{0}\right)^{2}}{\Re e \tau_{0}+\left|\tau_{0}\right|}\right)^{\frac{1}{2}}>0, \quad R_{0}=\frac{\Re e \tau_{0}}{\Im m \tau_{0}}-\operatorname{sign}\left(\Im m \tau_{0}\right) \sqrt{1+\left(\frac{\Re e \tau_{0}}{\Im m \tau_{0}}\right)^{2}} .
$$

By setting

$$
\begin{cases}\theta_{0}=\operatorname{atan} t_{0}, & \in] 0, \pi / 2[, \\ \mathcal{V}_{0}=\operatorname{sign}\left(R_{0}\right) \frac{t_{0}}{\sqrt{1+t_{0}^{2}}} \sqrt{X_{0}}, & \in \mathbb{R}, \\ \varepsilon_{0}=\mathcal{V}_{0} R_{0}=\left|R_{0}\right| \frac{t_{0}}{\sqrt{1+t_{0}^{2}}} \sqrt{X_{0}}, & >0,\end{cases}
$$

we have inverted the transformation (71), in other words:

$$
X_{0}=X\left(\theta_{0}, \mathcal{V}_{0}\right) \text { and } \tau_{0}=\tau\left(\theta_{0}, \varepsilon_{0}, \mathcal{V}_{0}\right)
$$

Therefore $g\left(X_{0}, \tau_{0}\right)$ implies $F_{p m l}\left(\mathcal{V}_{0}, \theta_{0}, \varepsilon_{0}\right)=0$, which means that (P1) in not true. 
Lemma 6 The property $\left(P_{1}\right)$ (or equivalently $\left(P_{2}\right)$ ) is realized if and only if the the condition $\left(\mathcal{C}_{2}\right)(i i)$ is satisfied as well as one of the two following conditions $\left(\mathcal{C}_{3}\right)_{1}$ or $\left(\mathcal{C}_{3}\right)_{1}$ :

$$
\begin{array}{ll}
\left(\mathcal{C}_{3}\right)_{1} & \left(c_{12}+c_{33}\right)^{2} \leq\left(c_{11}-c_{33}\right)\left(c_{22}-c_{33}\right), \\
\left(\mathcal{C}_{3}\right)_{2} & \left(c_{11}+c_{33}\right)\left(c_{12}+c_{33}\right)^{2} \geq\left(c_{11}-c_{33}\right)\left(c_{11} c_{22}-c_{33}^{2}\right) .
\end{array}
$$

Proof. Let us rewrite $G(X, \tau)$ as a polyomial of degree two with respect to $\tau$ :

$$
G(X, \tau)=\left(c_{33}-X\right)\left(c_{22}-X\right) \tau^{2}+\left(c_{11}\left(c_{22}-X\right)+c_{33}\left(c_{33}-X\right)-\left(c_{12}+c_{33}\right)^{2}\right) \tau+c_{11} c_{33} .
$$

Its discriminant is given by:

$$
\Delta(X)=\alpha_{2} X^{2}+2 \alpha_{1} X+\alpha_{0}
$$

with

$$
\left\{\begin{array}{l}
\alpha_{2}=\left(c_{11}-c_{33}\right)^{2} \geq 0 \\
\alpha_{1}=\left(c_{11}+c_{33}\right)\left(c_{12}+c_{33}\right)^{2}-\left(c_{11}-c_{33}\right)\left(c_{11} c_{22}-c_{33}^{2}\right) \\
\alpha_{0}=\left(c_{11} c_{22}-c_{12}^{2}\right)\left(c_{11} c_{22}-\left(c_{12}+2 c_{33}\right)^{2}\right)
\end{array}\right.
$$

Obviously, we have the equivalence

$$
\left(P_{2}\right) \Longleftrightarrow \Delta(X) \geq 0, \quad \forall X \geq 0
$$

This means that the two roots of $\Delta(X)$ are either non real either negative, which is equivalent to:

$$
\left.\alpha_{0} \geq 0 \quad \text { (i.e. }\left(\mathcal{C}_{2}\right)\right) \text { and (i) or (ii), }
$$

where

$$
\left\{\begin{array}{l}
(i) \quad \alpha_{0} \alpha_{2} \geq \alpha_{1}^{2}, \\
(i i) \quad \alpha_{1} \geq 0 \quad\left(\text { i.e. }\left(\mathcal{C}_{3}\right)_{2}\right) .
\end{array}\right.
$$

To conclude, it suffices to remark that the inequality (i) above is nothing but $\left(\mathcal{C}_{3}\right)_{1}$ since a tedious but simple computation shows that:

$$
\alpha_{1}^{2}-\alpha_{0} \alpha_{2}=4 c_{11} c_{33}\left(c_{12}+c_{33}\right)^{2}\left[\left(c_{12}+c_{33}\right)^{2}-\left(c_{22}-c_{33}\right)\left(c_{11}-c_{33}\right)\right] .
$$

As an immediate consequence, we state the:

Theorem 4 A sufficient condition for the stability of the PML system (26) with absorption in the $x_{1}$ direction is that conditions $\left(\mathcal{C}_{1}\right)$ and $\left(\mathcal{C}_{2}\right)$ be satisfied as well as one of the conditions $\left(\mathcal{C}_{3}\right)_{1}$ or $\left(\mathcal{C}_{3}\right)_{2}$. This is equivalent to saying that one of the two following conditions is realized:

$$
\begin{aligned}
& \left(\mathcal{C}_{x_{1}}\right)_{1} \quad\left(c_{12}+c_{33}\right)^{2}<\left(c_{11}-c_{33}\right)\left(c_{22}-c_{33}\right) \\
& \left(\mathcal{C}_{x_{1}}\right)_{2} \quad \begin{cases}\text { (i) } \quad\left(c_{11}-c_{33}\right)\left(c_{22}-c_{33}\right) \leq\left(c_{12}+c_{33}\right)^{2} \leq \max \left\{-c_{33}\left(c_{22}-c_{33}\right), c_{11}\left(c_{22}-c_{33}\right)\right\} \\
\text { (ii) } \quad\left(c_{11}-c_{33}\right)\left(c_{11} c_{22}-c_{33}^{2}\right)<\left(c_{11}+c_{33}\right)\left(c_{12}+c_{33}\right)^{2} \\
\text { (iii) } \quad\left(c_{12}+2 c_{33}\right)^{2}<c_{11} c_{22}\end{cases}
\end{aligned}
$$


Proof. It simply remains to explain why satisfying $\left(\mathcal{C}_{1}\right),\left(\mathcal{C}_{2}\right)$ and $\left(\mathcal{C}_{3}\right)_{1}$ or $\left(\mathcal{C}_{3}\right)_{2}$ is equivalent to $\left(\mathcal{C}_{x_{1}}\right)_{1}$ or $\left(\mathcal{C}_{x_{1}}\right)_{2}$. We first observe that the two real numbers:

$$
-c_{33}\left(c_{22}-c_{33}\right) \quad \text { and } \quad c_{11}\left(c_{22}-c_{33}\right)
$$

have opposite signs. As a consequence, the maximum of these two values is positive and greater than their sum:

$$
\left(c_{11}-c_{33}\right)\left(c_{22}-c_{33}\right) \leq \max \left\{-c_{33}\left(c_{22}-c_{33}\right), c_{11}\left(c_{22}-c_{33}\right)\right\} \quad(\geq 0) .
$$

We next observe that condition $\left(\mathcal{C}_{1}\right)$ means that $\left(c_{12}+c_{33}\right)^{2}$ belongs to the interval delimited by the same two numbers. Since $\left(c_{12}+c_{33}\right)^{2}>0$, this reduces to:

$$
\left(c_{12}+c_{33}\right)^{2} \leq \max \left\{-c_{33}\left(c_{22}-c_{33}\right), c_{11}\left(c_{22}-c_{33}\right)\right\} .
$$

As $-c_{33}\left(c_{22}-c_{33}\right) \leq c_{33}^{2}$ and $c_{11}\left(c_{22}-c_{33}\right) \leq c_{11} c_{22}$, we deduce that:

$$
(75) \quad \Longrightarrow \quad\left(c_{12}+c_{33}\right)^{2} \leq \max \left\{c_{33}^{2}, c_{11} c_{22}\right\} \leq c_{33}^{2}+c_{11} c_{22} \text {, }
$$

in other words:

$$
\left(\mathcal{C}_{1}\right) \Longrightarrow\left(\mathcal{C}_{2}\right)(i i)
$$

The less immediate remark, whose proof is left to the reader, is that:

$$
\left(c_{12}+c_{33}\right)^{2} \leq\left(c_{11}-c_{33}\right)\left(c_{22}-c_{33}\right) \quad \Longrightarrow \quad\left(c_{12}+2 c_{33}\right)^{2} \leq c_{11} c_{22} . \quad\left(\text { i. e. } \quad\left(\mathcal{C}_{2}\right)(i)\right)
$$

One then concludes as follows:

1. If $\left(\mathcal{C}_{x 1}\right)_{1}$ - which is nothing but $\left(\mathcal{C}_{3}\right)_{1}-\left(\mathcal{C}_{1}\right)$ is satisfied thanks to $(74)$ and $(75)$. Therefore $\left(\mathcal{C}_{2}\right)(i i)$ holds thanks to $(77)$ and $\left(\mathcal{C}_{2}\right)(i)$ holds thanks to $(76)$.

If $\left(\mathcal{C}_{x 1}\right)_{2}$ is satisfied, $\left(\mathcal{C}_{1}\right)$ (and thus $\left.\left(\mathcal{C}_{2}\right)(i i)\right)$ results from $\left(\mathcal{C}_{x 1}\right)_{2}(i),\left(\mathcal{C}_{1}\right)$ from $\left(\mathcal{C}_{x 1}\right)_{2}(i i i)$ and $\left(\mathcal{C}_{3}\right)_{2}$ from $\left(\mathcal{C}_{x 1}\right)_{2}(i i)$.

2. Reciprocally, if $\left(\mathcal{C}_{1}\right),\left(\mathcal{C}_{2}\right)$ and $\left(\mathcal{C}_{3}\right)_{1}$ or $\left(\mathcal{C}_{3}\right)_{2}$ are satisfied, then, according to (74) and (75) either

$$
\left(c_{12}+c_{33}\right)^{2}<\left(c_{11}-c_{33}\right)\left(c_{22}-c_{33}\right)
$$

in which case $\left(\mathcal{C}_{x 1}\right)_{1}$ holds, either

$$
\left(c_{11}-c_{33}\right)\left(c_{22}-c_{33}\right) \leq\left(c_{12}+c_{33}\right)^{2} \leq \max \left\{-c_{33}\left(c_{22}-c_{33}\right), c_{11}\left(c_{22}-c_{33}\right)\right\},
$$

that is $\left(\mathcal{C}_{x 1}\right)_{2}(i)$, in which case $\left(\mathcal{C}_{x 1}\right)_{2}(i)$ and $\left(\mathcal{C}_{x 1}\right)_{2}(i)$ result from $\left(\mathcal{C}_{2}\right)(i)$ and $\left(\mathcal{C}_{3}\right)_{2}$ (which is satisfied since $\left(\mathcal{C}_{3}\right)_{1}$ is not).

In order to obtain a sufficient condition for the stability for the PML model with absorption in the $x_{2}$ direction, we simply have to permute $c_{11}$ and $c_{22}$ :

Theorem 5 The PML system (26) with absorption in the $x_{2}$ direction is stable as soon as one of the two following conditions is realized:

$$
\begin{aligned}
\left(\mathcal{C}_{x_{2}}\right)_{1} & \left(c_{12}+c_{33}\right)^{2}<\left(c_{11}-c_{33}\right)\left(c_{22}-c_{33}\right), \\
\left(\mathcal{C}_{x_{2}}\right)_{2} & \begin{cases}\text { (i) } \quad & \left(c_{11}-c_{33}\right)\left(c_{22}-c_{33}\right) \leq\left(c_{12}+c_{33}\right)^{2} \leq \max \left\{-c_{33}\left(c_{22}-c_{33}\right), c_{11}\left(c_{22}-c_{33}\right)\right\} \\
\text { (ii) } \quad & \left(c_{22}-c_{33}\right)\left(c_{11} c_{22}-c_{33}^{2}\right)<\left(c_{22}+c_{33}\right)\left(c_{12}+c_{33}\right)^{2}, \\
\text { (iii) } & \left(c_{12}+2 c_{33}\right)^{2}<c_{11} c_{22} .\end{cases}
\end{aligned}
$$

INRIA 
Remark 3 In the isotropic case, the coefficients are expressed in terms of Lamé's coefficients $\lambda, \mu$ through relation (8) one easily checks that condition $\left(\mathcal{C}_{1}\right)_{x_{1}} \equiv\left(\mathcal{C}_{1}\right)_{x_{2}}$ is obviously satisfied, therefore the $P M L$ model with absorption in $x_{1}$ or in $x_{2}$ is stable for any isotropic material.

Remark 4 The conditions (7) on the coefficients do not imply the positivity of coefficient $c_{12}$. However, in the applications this coefficient is often positive. It is easy to see that in this case, if $c_{33}>c_{22}$, the condition $\left(\mathcal{C}_{1}\right)$ can not be satisfied, therefore the PML model with absorption in the $x_{1}$ direction is not stable. In the same way, if $c_{33}>c_{11}$, the PML model with absorption in the $x_{2}$ direction is not stable. Therefore, if $c_{12} \geq 0$, a necessary condition for the general PML model to be stable is that $c_{33} \leq c_{22}$ and $c_{33} \leq c_{11}$.

\subsection{Back to the numerical experiments of section 3.4.3}

We come back to the numerical experiments done at section 3.4.3. We have seen that the material $(\mathrm{V})$ satisfies the geometrical stability condition. We can check that it does not satisfy the necessary condition $\left(\mathcal{C}_{2}\right)$ which is the high frequency condition in the vicinity of the mode 0 of order 4 . On the other hand the condition $\left(\mathcal{C}_{3}\right)$ is satisfied, which means that the branches issued from the physical modes always stay in the good side of the complex plane. Therefore, the instability appearing in the experiment (V)-1, observed in figure 21 , can be explained by the fact that $\left(\mathcal{C}_{2}\right)$ is not satisfied. One can notice that this instability appears after a very long time, so that in practice, it does not seem to be a significant problem.

In the experiment $(\mathrm{V})-2$, we added a layer in the $x_{2}$ direction, and this time one can check that the condition $\left(\mathcal{C}_{3}\right)$ written for the $x_{2}$ layer is not satisfied, which means that there exists some branches that cross the real axis. Since the instability observed in figure 22 appears much sooner than in the experiment (V)-1, we think that it is due to the fact that the branches issued from the physical modes are in the bad side of the complex plane for some frequencies.

Conjecture. We conjecture that the sufficient conditions of theorems 4 and 5 are also necessary stability conditions. The idea is that, as soon as $\left(\mathcal{C}_{3}\right)_{1}$ or $\left(\mathcal{C}_{3}\right)_{2}$ is not satisfied, there exists $\left.\theta_{0} \in\right] 0, \pi / 2[$ such that one curve $\varepsilon \mapsto\left(\mathcal{V}_{\theta_{0}}\right)_{j}(\varepsilon)$ meets the real axis for some $\varepsilon=\varepsilon_{0}>0$. The proof would be complete if one would be able to show that this curve really crosses the real axis at $\varepsilon=\varepsilon_{0}$ (case 2 of Figure 23). This would essentially correspond to proving that:

$$
\Im m\left\{\frac{\partial}{\partial \varepsilon}\left(\mathcal{V}_{\theta_{0}}\right)_{j}\left(\varepsilon_{0}\right)\right\} \neq 0
$$

that we have not been able to do... Another element for this conjecture is related to the fact that the
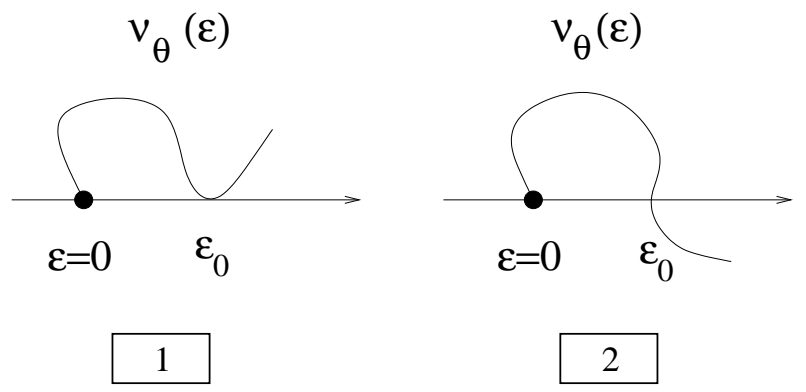

Figure 23: The two possibilities for each curve $\mathcal{V}_{\theta}(\varepsilon)$.

instability observed in the experiment $(\mathrm{V})-2$, in figure 22 , is due to the fact that the condition $\left(\mathcal{C}_{3}\right)$ is not satisfied.

$\mathrm{RR} \mathrm{n}^{\circ} 4304$ 


\section{References}

[1] S. Abarbanel and D. Gottlieb. A mathematical analysis of the PML method. J. Comput. Phys, 134(2):357-363, 1997.

[2] B. A. Auld. Acoustic Fields and Elastic Waves in Solids, volume I et II. Wiley, 1973.

[3] E. Bécache and P. Joly. On the analysis of Bérenger's Perfectly Matched Layers for Maxwell equations. Rapport de Recherche 4164, INRIA., 2001.

[4] J. P. Bérenger. A Perfectly Matched Layer for the Absorption of Electromagnetic Waves. J. of Comp. Phys., 114:185-200, 1994.

[5] J.P. Bérenger. Three-dimensional perfectly matched layer for the absorption of electromagnetic waves. J. Comput. Phys., 127(2):363-379, 1996.

[6] J.P. Bérenger. Improved PML for the FDTD Solution of Wave-Structure Interaction Problems . IEEE transactions on Antennas and Propagation., 45(3):466-473, march 1997.

[7] W. C. Chew and W. H. Weedon. A 3D Perfectly Matched Medium from Modified Maxwell's Equations with stretched coordinates. IEEE microwave and Optical Tech. Letters, 7(13):599-604, 1994.

[8] G. Cohen and S. Fauqueux. Mixed Finite Elements with Mass-Lumping for the Transient Wave Equation. J. of Comp. Acous., 8:171-188, 2000.

[9] F. Collino. Perfectly Matched Absorbing Layers for the Paraxial Equations . J. of Comp. Phys., 131(1):164-180, 1997.

[10] F. Collino and P. Monk. Conditions et couches absorbantes pour les équations de Maxwell. In G. Cohen and P. Joly, editors, Aspects récents en méthodes numériques pour les équations de Maxwell, Ecole des Ondes, chapter 4. INRIA, Rocquencourt, 1998.

[11] F. Collino and C. Tsogka. Application of the pml absorbing layer model to the linear elastodynamic problem in anisotropic heteregeneous media. Geophysics, 66(1):294-307, 2001.

[12] T. Fouquet. Personal communication.

[13] F. Hastings, J.B. Schneider, and S. L. Broschat. Application of the perfectly matched layer (PML) absorbing boundary condition to elastic wave propagation. J. Acoust. Soc. Am., 100:3061-3069, 1996.

[14] J. S. Hesthaven. On the Analysis and Construction of Perfectly Matched Layers for the Linearized Euler Equations. J. Comp. Phys., 142:129-147, 1998.

[15] F. Q. Hu. On absorbing boundary conditions for linearized euler equations by a perfectly matched layer. J. Comp. Phys., 129:201-219, 1996.

[16] H-O. Kreiss and J. Lorenz. Initial-Boundary Value Problems and the Navier-Stokes Equations. In Pure and Appl. Math., volume 136. Academic Press, Boston, USA, 1989.

[17] J. Métral and O. Vacus. Caractère bien posé du problème de Cauchy pour le système de Bérenger. C.R.A.S., I Math.(10):847-852, 1999.

[18] P. G. Petropoulos, L. Zhao, and A. C. Cangellaris. A reflectionless sponge layer absorbing boundary condition for the solution of Maxwell's equations with high-order staggered finite difference schemes. J. Comput. Phys., 139(1):184-208, 1998. 
[19] A. N. Rahmouni. Des modèles PML bien posés pour divers problèmes hyperboliques. PhD thesis, Université Paris Nord-Paris XIII, 2000.

[20] C.M. Rappaport. Perfectly Matched Absorbing Conditions Based on Anisotropic Lossy Mapping of Space. IEEE Microwave and Guided Wave Letters., 5(3):90-92, 1995.

[21] C. K. W. Tam, L. Auriault, and F. Cambuli. Perfectly matched layer as an absorbing boundary condition for the linearized euler equations in open and ducted domains. J. Comput. Phys., 144(1):213-234, 1998.

[22] F. L. Teixeira and W. C. Chew. Analytical derivation of a conformal perfectly matched absorber for electromagnetic waves. Micro. Opt. Tech. Lett., 17:231-236, 1998.

[23] E. Turkel and A. Yefet. Absorbing PML boundary layers for wave-like equations. Appl. Numer. Math., 27(4):533-557, 1998.

[24] L. Zhao and A. C. Cangellaris. A General Approach for the Development of Unsplit-Field TimeDomain Implementations of Perfectly Matched Layers for FDTD Grid Truncation. IEEE Microwave and Guided Letters, 6(5), May 1996.

[25] L. Zhao and A.C. Cangellaris. GT PML: Generalize Theory of Perfectly Matched Layers and Its Application to the Reflectionless Truncation of Finite-Difference Time-Domain Grids. IEEE Trans. Microwave Theory Tech., 44:2555-2563, 1996. 


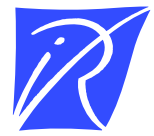

Unité de recherche INRIA Rocquencourt Domaine de Voluceau - Rocquencourt - BP 105 - 78153 Le Chesnay Cedex (France)

Unité de recherche INRIA Lorraine : LORIA, Technopôle de Nancy-Brabois - Campus scientifique 615, rue du Jardin Botanique - BP 101 - 54602 Villers-lès-Nancy Cedex (France)

Unité de recherche INRIA Rennes : IRISA, Campus universitaire de Beaulieu - 35042 Rennes Cedex (France)

Unité de recherche INRIA Rhône-Alpes : 655, avenue de l'Europe - 38330 Montbonnot-St-Martin (France)

Unité de recherche INRIA Sophia Antipolis : 2004, route des Lucioles - BP 93 - 06902 Sophia Antipolis Cedex (France)

INRIA - Domaine de Voluceau - Rocquencourt, BP 105 - 78153 Le Chesnay Cedex (France)

http://www.inria.fr

ISSN 0249-6399 